\title{
Lax representation of the hyperbolic van Diejen dynamics with two coupling parameters
}

\author{
B.G. PUSZTAI ${ }^{a, b}$ and T.F. GÖRBE ${ }^{c}$ \\ ${ }^{a}$ Bolyai Institute, University of Szeged, \\ Aradi vértanúk tere 1, H-6720 Szeged, Hungary \\ ${ }^{b}$ MTA Lendület Holographic QFT Group, Wigner RCP, \\ H-1525 Budapest 114, P.O.B. 49, Hungary \\ e-mail: gpusztai@math.u-szeged.hu \\ ${ }^{c}$ Department of Theoretical Physics, University of Szeged, \\ Tisza Lajos krt 84-86, H-6720 Szeged, Hungary \\ e-mail: tfgorbe@physx.u-szeged.hu
}

\begin{abstract}
In this paper, we construct a Lax pair for the classical hyperbolic van Diejen system with two independent coupling parameters. Built upon this construction, we show that the dynamics can be solved by a projection method, which in turn allows us to initiate the study of the scattering properties. As a consequence, we prove the equivalence between the first integrals provided by the eigenvalues of the Lax matrix and the family of van Diejen's commuting Hamiltonians. Also, at the end of the paper, we propose a candidate for the Lax matrix of the hyperbolic van Diejen system with three independent coupling constants.
\end{abstract}

Keywords: Integrable systems; Ruijsenaars-Schneider-van Diejen models; Lax matrices MSC (2010): 70E40, 70G65, 70H06

PACS number: 02.30.Ik

\section{Contents}

1 Introduction $\quad 2$

2 Preliminaries from group theory

3 Algebraic properties of the Lax matrix 5

3.1 The matrix $L$ and the Lie group $U(n, n) \ldots \ldots \ldots \ldots$

3.2 Commutation relation and regularity .................... 9

4 Analyzing the dynamics $\quad \mathbf{1 3}$

4.1 Completeness of the Hamiltonian vector field . . . . . . . . . . . . . . . . 14

4.2 Dynamics of the vector $F \ldots \ldots \ldots \ldots \ldots$

4.3 Lax representation of the dynamics . . . . . . . . . . . . . . . . 19

4.4 Geodesic interpretation . . . . . . . . . . . . . . . . . . . . . . . . . . . .

4.5 Temporal asymptotics . . . . . . . . . . . . . . . . 27 
5 Spectral invariants of the Lax matrix 29

5.1 Link to the 5 -parameter family of van Diejen systems . . . . . . . . . . 29

5.2 Poisson brackets of the eigenvalues of $L \ldots \ldots \ldots \ldots \ldots$

6 Discussion

\section{Introduction}

The Ruijsenaars-Schneider-van Diejen (RSvD) systems, or simply van Diejen systems [1, 2, 3, are multi-parametric integrable deformations of the translation invariant Ruijsenaars-Schneider (RS) models [4, 5]. Moreover, in the so-called 'non-relativistic' limit, they reproduce the Calogero-Moser-Sutherland (CMS) models [6, 7, 8, 9, associated with the $B C$-type root systems. However, compared to the translation invariant $A$-type models, the geometrical picture underlying the most general classical van Diejen models is far less developed. The most probable explanation of this fact is the lack of Lax representation for the van Diejen dynamics. For this reason, working mainly in a symplectic reduction framework, in the last couple of years we undertook the study of the $B C$-type rational van Diejen models [10, 11, 12, 13, 14, 15]. By going one stage up, in this paper we wish to report on our first results about the hyperbolic variants of the van Diejen family.

In order to describe the Hamiltonian systems of our interest, let us recall that the configuration space of the hyperbolic $n$-particle van Diejen model is the open subset

$$
Q=\left\{\lambda=\left(\lambda_{1}, \ldots, \lambda_{n}\right) \in \mathbb{R}^{n} \mid \lambda_{1}>\ldots>\lambda_{n}>0\right\} \subseteq \mathbb{R}^{n},
$$

that can be seen as an open Weyl chamber of type $B C_{n}$. The cotangent bundle of $Q$ is trivial, and it can be naturally identified with the open subset

$$
P=Q \times \mathbb{R}^{n}=\left\{(\lambda, \theta)=\left(\lambda_{1}, \ldots, \lambda_{n}, \theta_{1}, \ldots, \theta_{n}\right) \in \mathbb{R}^{2 n} \mid \lambda_{1}>\ldots>\lambda_{n}>0\right\} \subseteq \mathbb{R}^{2 n} .
$$

Following the widespread custom, throughout the paper we shall occasionally think of the letters $\lambda_{a}$ and $\theta_{a}(1 \leq a \leq n)$ as globally defined coordinate functions on $P$. For example, using this latter interpretation, the canonical symplectic form on the phase space $P \cong T^{*} Q$ can be written as

$$
\omega=\sum_{c=1}^{n} \mathrm{~d} \lambda_{c} \wedge \mathrm{d} \theta_{c}
$$

whereas the fundamental Poisson brackets take the form

$$
\left\{\lambda_{a}, \lambda_{b}\right\}=0, \quad\left\{\theta_{a}, \theta_{b}\right\}=0, \quad\left\{\lambda_{a}, \theta_{b}\right\}=\delta_{a, b} \quad(1 \leq a, b \leq n) .
$$

The principal goal of this paper is to study the dynamics generated by the smooth Hamiltonian function

$$
H=\sum_{a=1}^{n} \cosh \left(\theta_{a}\right)\left(1+\frac{\sin (\nu)^{2}}{\sinh \left(2 \lambda_{a}\right)^{2}}\right)^{\frac{1}{2}} \prod_{\substack{c=1 \\(c \neq a)}}^{n}\left(1+\frac{\sin (\mu)^{2}}{\sinh \left(\lambda_{a}-\lambda_{c}\right)^{2}}\right)^{\frac{1}{2}}\left(1+\frac{\sin (\mu)^{2}}{\sinh \left(\lambda_{a}+\lambda_{c}\right)^{2}}\right)^{\frac{1}{2}}
$$


where $\mu, \nu \in \mathbb{R}$ are arbitrary coupling constants satisfying the conditions

$$
\sin (\mu) \neq 0 \neq \sin (\nu) .
$$

Note that $H$ (1.5) does belong to the family of the hyperbolic $n$-particle van Diejen Hamiltonians with two independent parameters $\mu$ and $\nu$ (cf. (5.11)). Of course, the values of the parameters $\mu$ and $\nu$ really matter only modulo $\pi$.

Now, we briefly outline the content of the paper. In the subsequent section, we start with a short overview on some relevant facts and notations from Lie theory. Having equipped with the necessary background material, in Section 3 we define our Lax matrix (3.6) for the van Diejen system (1.5), and also investigate its main algebraic properties. In Section 4 we turn to the study of the Hamiltonian flow generated by (1.5). As the first step, in Theorem 5 we formulate the completeness of the corresponding Hamiltonian vector field. Most importantly, in Theorem 8 we provide a Lax representation of the dynamics, whereas in Theorem 12 we establish a solution algorithm of purely algebraic nature. Making use of the projection method formulated in Theorem 12, we also initiate the study of the scattering properties of the system (1.5). Our rigorous results on the temporal asymptotics of the maximally defined trajectories are summarized in Lemma 13. Section 5 serves essentially two purposes. In Subsection 5.1] we elaborate the link between our special 2-parameter family of Hamiltonians (1.5) and the most general 5-parameter family of hyperbolic van Diejen systems (5.5). At the level of the coupling parameters the relationship can be read off from the equation (5.8). Furthermore, in Lemma 14 we affirm the equivalence between van Diejen's commuting family of Hamiltonians and the coefficients of the characteristic polynomial of the Lax matrix (3.6). Based on this technical result, in Theorem 15] we can infer that the eigenvalues of the proposed Lax matrix (3.6) provide a commuting family of first integrals for the Hamiltonian system (1.5). We conclude the paper with Section 6, where we discuss the potential applications, and also offer some open problems and conjectures. In particular, in (6.5) we propose a Lax matrix for the 3-parameter family of hyperbolic van Diejen systems defined in (6.7).

\section{Preliminaries from group theory}

This section has two main objectives. Besides fixing the notations used throughout the paper, we also provide a brief account on some relevant facts from Lie theory underlying our study of the 2-parameter family of hyperbolic van Diejen systems (1.5). For convenience, our conventions closely follow Knapp's book [16].

As before, by $n \in \mathbb{N}=\{1,2, \ldots\}$ we denote the number of particles. Let $N=2 n$, and also introduce the shorthand notations

$$
\mathbb{N}_{n}=\{1, \ldots, n\} \text { and } \mathbb{N}_{N}=\{1, \ldots, N\} .
$$

With the aid of the $N \times N$ matrix

$$
C=\left[\begin{array}{ll}
0_{n} & \mathbf{1}_{n} \\
\mathbf{1}_{n} & 0_{n}
\end{array}\right]
$$

we define the non-compact real reductive matrix Lie group

$$
G=U(n, n)=\left\{y \in G L(N, \mathbb{C}) \mid y^{*} C y=C\right\},
$$


in which the set of unitary elements

$$
K=\left\{y \in G \mid y^{*} y=\mathbf{1}_{N}\right\} \cong U(n) \times U(n)
$$

forms a maximal compact subgroup. The Lie algebra of $G(2.3)$ takes the form

$$
\mathfrak{g}=\mathfrak{u}(u, n)=\left\{Y \in \mathfrak{g l}(N, \mathbb{C}) \mid Y^{*} C+C Y=0\right\},
$$

whereas for the Lie subalgebra corresponding to $K(2.4)$ we have the identification

$$
\mathfrak{k}=\left\{Y \in \mathfrak{g} \mid Y^{*}+Y=0\right\} \cong \mathfrak{u}(n) \oplus \mathfrak{u}(n) .
$$

Upon introducing the subspace

$$
\mathfrak{p}=\left\{Y \in \mathfrak{g} \mid Y^{*}=Y\right\},
$$

we can write the decomposition $\mathfrak{g}=\mathfrak{k} \oplus \mathfrak{p}$, which is orthogonal with respect to the usual trace pairing defined on the matrix Lie algebra $\mathfrak{g}$. Let us note that the restriction of the exponential map onto the complementary subspace $\mathfrak{p}$ (2.7) is injective. Moreover, the image of $\mathfrak{p}$ under the exponential map can be identified with the set of the positive definite elements of the group $U(n, n)$; that is,

$$
\exp (\mathfrak{p})=\{y \in U(n, n) \mid y>0\} .
$$

Notice that, due to the Cartan decomposition $G=\exp (\mathfrak{p}) K$, the above set can be also naturally identified with the non-compact symmetric space associated with the pair $(G, K)$, i.e.,

$$
\exp (\mathfrak{p}) \cong U(n, n) /(U(n) \times U(n)) \cong S U(n, n) / S(U(n) \times U(n)) .
$$

To get a more detailed picture about the structure of the reductive Lie group $U(n, n)$, in $\mathfrak{p}$ (2.7) we introduce the maximal Abelian subspace

$$
\mathfrak{a}=\left\{X=\operatorname{diag}\left(x_{1}, \ldots, x_{n},-x_{1}, \ldots,-x_{n}\right) \mid x_{1}, \ldots, x_{n} \in \mathbb{R}\right\}
$$

Let us recall that we can attain every element of $\mathfrak{p}$ by conjugating the elements of $\mathfrak{a}$ with the elements of the compact subgroup $K(2.4)$. More precisely, the map

$$
\mathfrak{a} \times K \ni(X, k) \mapsto k X k^{-1} \in \mathfrak{p}
$$

is well-defined and onto. As for the centralizer of $\mathfrak{a}$ inside $K(2.4)$, it turns out to be the Abelian Lie group

$$
M=Z_{K}(\mathfrak{a})=\left\{\operatorname{diag}\left(e^{\mathrm{i} \chi_{1}}, \ldots, e^{\mathrm{i} \chi_{n}}, e^{\mathrm{i} \chi_{1}}, \ldots, e^{\mathrm{i} \chi_{n}}\right) \mid \chi_{1}, \ldots, \chi_{n} \in \mathbb{R}\right\}
$$

with Lie algebra

$$
\mathfrak{m}=\left\{\operatorname{diag}\left(\mathrm{i} \chi_{1}, \ldots, \mathrm{i} \chi_{n}, \mathrm{i} \chi_{1}, \ldots, \mathrm{i} \chi_{n}\right) \mid \chi_{1}, \ldots, \chi_{n} \in \mathbb{R}\right\}
$$

Let $\mathfrak{m}^{\perp}$ and $\mathfrak{a}^{\perp}$ denote the sets of the off-diagonal elements in the subspaces $\mathfrak{k}$ and $\mathfrak{p}$, respectively; then clearly we can write the refined orthogonal decomposition

$$
\mathfrak{g}=\mathfrak{m} \oplus \mathfrak{m}^{\perp} \oplus \mathfrak{a} \oplus \mathfrak{a}^{\perp}
$$


To put it simple, each Lie algebra element $Y \in \mathfrak{g}$ can be decomposed as

$$
Y=Y_{\mathfrak{m}}+Y_{\mathfrak{m}^{\perp}}+Y_{\mathfrak{a}}+Y_{\mathfrak{a}^{\perp}}
$$

with unique components belonging to the subspaces indicated by the subscripts.

Throughout our work the commuting family of linear operators

$$
\operatorname{ad}_{X}: \mathfrak{g l}(N, \mathbb{C}) \rightarrow \mathfrak{g l}(N, \mathbb{C}), \quad Y \mapsto[X, Y]
$$

defined for the diagonal matrices $X \in \mathfrak{a}$ plays a distinguished role. Let us note that the (real) subspace $\mathfrak{m}^{\perp} \oplus \mathfrak{a}^{\perp} \subseteq \mathfrak{g l}(N, \mathbb{C})$ is invariant under $\operatorname{ad}_{X}$, whence the restriction

$$
\widetilde{\operatorname{ad}}_{X}=\left.\operatorname{ad}_{X}\right|_{\mathfrak{m}^{\perp} \oplus \mathfrak{a}^{\perp}} \in \mathfrak{g l}\left(\mathfrak{m}^{\perp} \oplus \mathfrak{a}^{\perp}\right)
$$

is a well-defined operator for each $X=\operatorname{diag}\left(x_{1}, \ldots, x_{n},-x_{1}, \ldots,-x_{n}\right) \in \mathfrak{a}$ with spectrum

$$
\operatorname{Spec}\left(\widetilde{\operatorname{ad}}_{X}\right)=\left\{x_{a}-x_{b}, \pm\left(x_{a}+x_{b}\right), \pm 2 x_{c} \mid a, b, c \in \mathbb{N}_{n}, a \neq b\right\} \text {. }
$$

Now, recall that the regular part of the Abelian subalgebra $\mathfrak{a}(2.10)$ is defined by the subset

$$
\mathfrak{a}_{\mathrm{reg}}=\left\{X \in \mathfrak{a} \mid \widetilde{\operatorname{ad}}_{X} \text { is invertible }\right\},
$$

in which the standard open Weyl chamber

$$
\mathfrak{c}=\left\{X=\operatorname{diag}\left(x_{1}, \ldots, x_{n},-x_{1}, \ldots,-x_{n}\right) \in \mathfrak{a} \mid x_{1}>\ldots>x_{n}>0\right\}
$$

is a connected component. Let us observe that it can be naturally identified with the configuration space $Q$ (1.1); that is, $Q \cong \mathfrak{c}$. Finally, let us recall that the regular part of $\mathfrak{p}(2.7)$ is defined as

$$
\mathfrak{p}_{\text {reg }}=\left\{k X k^{-1} \in \mathfrak{p} \mid X \in \mathfrak{a}_{\text {reg }} \text { and } k \in K\right\} .
$$

As a matter of fact, from the map (2.11) we can derive a particularly useful characterization for the open subset $\mathfrak{p}_{\text {reg }} \subseteq \mathfrak{p}$. Indeed, the map

$$
\mathfrak{c} \times(K / M) \ni(X, k M) \mapsto k X k^{-1} \in \mathfrak{p}_{\text {reg }}
$$

turns out to be a diffeomorphism, providing the identification $\mathfrak{p}_{\text {reg }} \cong \mathfrak{c} \times(K / M)$.

\section{Algebraic properties of the Lax matrix}

Having reviewed the necessary notions and notations from Lie theory, in this section we propose a Lax matrix for the hyperbolic van Diejen system of our interest (1.5). To make the presentation simpler, with any $\lambda=\left(\lambda_{1}, \ldots, \lambda_{n}\right) \in \mathbb{R}^{n}$ and $\theta=\left(\theta_{1}, \ldots, \theta_{n}\right) \in \mathbb{R}^{n}$ we associate the real $N$-tuples

$$
\Lambda=\left(\lambda_{1}, \ldots, \lambda_{n},-\lambda_{1}, \ldots,-\lambda_{n}\right) \text { and } \Theta=\left(\theta_{1}, \ldots, \theta_{n},-\theta_{1}, \ldots,-\theta_{n}\right),
$$


respectively, and also define the $N \times N$ diagonal matrix

$$
\boldsymbol{\Lambda}=\operatorname{diag}\left(\Lambda_{1}, \ldots, \Lambda_{N}\right)=\operatorname{diag}\left(\lambda_{1}, \ldots, \lambda_{n},-\lambda_{1}, \ldots,-\lambda_{n}\right) \in \mathfrak{a} .
$$

Notice that if $\lambda \in \mathbb{R}^{n}$ is a regular element in the sense that the corresponding diagonal matrix $\Lambda$ (3.2) belongs to $\mathfrak{a}_{\text {reg }}(2.19)$, then for each $j \in \mathbb{N}_{N}$ the complex number

$$
z_{j}=-\frac{\sinh \left(\mathrm{i} \nu+2 \Lambda_{j}\right)}{\sinh \left(2 \Lambda_{j}\right)} \prod_{\substack{c=1 \\(c \neq j, j-n)}}^{n} \frac{\sinh \left(\mathrm{i} \mu+\Lambda_{j}-\lambda_{c}\right)}{\sinh \left(\Lambda_{j}-\lambda_{c}\right)} \frac{\sinh \left(\mathrm{i} \mu+\Lambda_{j}+\lambda_{c}\right)}{\sinh \left(\Lambda_{j}+\lambda_{c}\right)}
$$

is well-defined. Thinking of $z_{j}$ as a function of $\lambda$, let us observe that its modulus $u_{j}=\left|z_{j}\right|$ takes the form

$$
u_{j}=\left(1+\frac{\sin (\nu)^{2}}{\sinh \left(2 \Lambda_{j}\right)^{2}}\right)^{\frac{1}{2}} \prod_{\substack{c=1 \\ c \neq j, j-n)}}^{n}\left(1+\frac{\sin (\mu)^{2}}{\sinh \left(\Lambda_{j}-\lambda_{c}\right)^{2}}\right)^{\frac{1}{2}}\left(1+\frac{\sin (\mu)^{2}}{\sinh \left(\Lambda_{j}+\lambda_{c}\right)^{2}}\right)^{\frac{1}{2}}
$$

and the property $z_{n+a}=\bar{z}_{a}\left(a \in \mathbb{N}_{n}\right)$ is also clear. Next, built upon the functions $z_{j}$ and $u_{j}$, we introduce the column vector $F \in \mathbb{C}^{N}$ with components

$$
F_{a}=e^{\frac{\theta_{a}}{2}} u_{a}^{\frac{1}{2}} \quad \text { and } \quad F_{n+a}=e^{-\frac{\theta_{a}}{2}} \bar{z}_{a} u_{a}^{-\frac{1}{2}} \quad\left(a \in \mathbb{N}_{n}\right) .
$$

At this point we are in a position to define our Lax matrix $L \in \mathfrak{g l}(N, \mathbb{C})$ with the entries

$$
L_{k, l}=\frac{\mathrm{i} \sin (\mu) F_{k} \bar{F}_{l}+\mathrm{i} \sin (\mu-\nu) C_{k, l}}{\sinh \left(\mathrm{i} \mu+\Lambda_{k}-\Lambda_{l}\right)} \quad\left(k, l \in \mathbb{N}_{N}\right) .
$$

Note that the matrix valued function $L$ is well-defined at each point $(\lambda, \theta) \in \mathbb{R}^{N}$ satisfying the regularity condition $\Lambda \in \mathfrak{a}_{\text {reg. }}$. Since $\mathfrak{c} \subseteq \mathfrak{a}_{\text {reg }}(2.20), L$ makes sense at each point of the phase space $P$ (1.2) as well. To give a motivation for the definition of $L=L(\lambda, \theta ; \mu, \nu)$ (3.6), let us observe that in its 'rational limit' we get back the Lax matrix of the rational van Diejen system with two parameters. Indeed, up to some irrelevant numerical factors caused by a slightly different convention, in the $\alpha \rightarrow 0^{+}$limit the matrix $L(\alpha \lambda, \theta ; \alpha \mu, \alpha \nu)$ tends to the rational Lax matrix $\mathcal{A}=\mathcal{A}(\lambda, \theta ; \mu, \nu)$ as defined in the equations (4.2)-(4.5) of paper [10]. In [10] we saw that $\mathcal{A}$ has many peculiar algebraic properties, that we wish to generalize for the proposed hyperbolic Lax matrix $L$ in the rest of this section.

\subsection{The matrix $L$ and the Lie group $U(n, n)$}

By inspecting the matrix entries (3.6), it is obvious that $L$ is Hermitian. However, it is a less trivial fact that $L$ is closely tied with the non-compact Lie group $U(n, n)(2.3)$. The purpose of this subsection is to explore this surprising relationship.

Proposition 1. The matrix $L$ (3.6) obeys the quadratic equation $L C L=C$. In other words, the matrix valued function $L$ takes values in the Lie group $U(n, n)$. 
Proof. Take an arbitrary element $(\lambda, \theta) \in \mathbb{R}^{N}$ satisfying the regularity condition $\Lambda \in \mathfrak{a}_{\text {reg. }}$ We start by observing that for each $a \in \mathbb{N}_{n}$ the complex conjugates of $z_{a}$ (3.3) and $F_{n+a}$ (3.5) can be obtained by changing the sign of the single component $\lambda_{a}$ of $\lambda$. Therefore, if $a, b \in \mathbb{N}_{n}$ are arbitrary indices, then by interchanging the components $\lambda_{a}$ and $\lambda_{b}$ of the $n$-tuple $\lambda$, the expression $(L C L)_{a, b} F_{a}^{-1} \bar{F}_{b}^{-1}$ readily transforms into $(L C L)_{n+a, n+b} F_{n+a}^{-1} \bar{F}_{n+b}^{-1}$. We capture this fact by writing

$$
\frac{(L C L)_{a, b}}{F_{a} \bar{F}_{b}} \underset{\lambda_{a} \leftrightarrow \lambda_{b}}{\rightsquigarrow} \frac{(L C L)_{n+a, n+b}}{F_{n+a} \bar{F}_{n+b}} \quad\left(a, b \in \mathbb{N}_{n}\right) .
$$

Similarly, if $a \neq b$, then from $(L C L)_{a, b} F_{a}^{-1} \bar{F}_{b}^{-1}$ we can recover $(L C L)_{n+a, b} F_{n+a}^{-1} \bar{F}_{b}^{-1}$ by exchanging $\lambda_{a}$ for $-\lambda_{a}$. Schematically, we have

$$
\frac{(L C L)_{a, b}}{F_{a} \bar{F}_{b}} \underset{\lambda_{a} \leftrightarrow-\lambda_{a}}{\rightsquigarrow} \frac{(L C L)_{n+a, b}}{F_{n+a} \bar{F}_{b}} \quad\left(a, b \in \mathbb{N}_{n}, a \neq b\right) .
$$

Furthermore, the expression $(L C L)_{a, b} F_{a}^{-1} \bar{F}_{b}^{-1}$ reproduces $(L C L)_{a, n+b} F_{a}^{-1} \bar{F}_{n+b}^{-1}$ upon swapping $\lambda_{b}$ for $-\lambda_{b}$, i.e.,

$$
\frac{(L C L)_{a, b}}{F_{a} \bar{F}_{b}} \underset{\lambda_{b} \leftrightarrow-\lambda_{b}}{\rightsquigarrow} \frac{(L C L)_{a, n+b}}{F_{a} \bar{F}_{n+b}} \quad\left(a, b \in \mathbb{N}_{n}, a \neq b\right) .
$$

Finally, the relationship between the remaining entries is given by the exchange

$$
(L C L)_{a, n+a} \underset{\lambda_{a} \leftrightarrow-\lambda_{a}}{\rightsquigarrow}(L C L)_{n+a, a} \quad\left(a \in \mathbb{N}_{n}\right) .
$$

The message of the above equations (3.7)-(3.10) is quite evident. Indeed, in order to prove the desired matrix equation $L C L=C$, it does suffice to show that $(L C L)_{a, b}=0$ for all $a, b \in \mathbb{N}_{n}$, and also that $(L C L)_{a, n+a}=1$ for all $a \in \mathbb{N}_{n}$.

Recalling the formulae (3.5) and (3.6) , it is clear that for all $a \in \mathbb{N}_{n}$ we can write

$$
\frac{(L C L)_{a, a}}{F_{a} \bar{F}_{a}}=2 \operatorname{Re}\left(\frac{\mathrm{i} \sin (\mu) z_{a}+\mathrm{i} \sin (\mu-\nu)}{\sinh \left(\mathrm{i} \mu+2 \lambda_{a}\right)}-\sum_{\substack{c=1 \\(c \neq a)}}^{n} \frac{\sin (\mu)^{2} z_{c}}{\sinh \left(\mathrm{i} \mu+\lambda_{a}+\lambda_{c}\right) \sinh \left(\mathrm{i} \mu-\lambda_{a}+\lambda_{c}\right)}\right) .
$$

To proceed further, we introduce a complex valued function $f_{a}$ depending on a single complex variable $w$ obtained simply by replacing $\lambda_{a}$ with $\lambda_{a}+w$ in the right-hand side of the above equation (3.11). Remembering (3.3), it is obvious that the resulting function is meromorphic with at most first order poles at the points

$$
w \equiv-\lambda_{a}, w \equiv \pm \mathrm{i} \mu / 2-\lambda_{a}, w \equiv \Lambda_{j}-\lambda_{a}\left(j \in \mathbb{N}_{N}\right) \quad(\bmod \mathrm{i} \pi)
$$

However, by inspecting the terms appearing in the explicit expression of $f_{a}$, a straightforward computation reveals immediately that the residue of $f_{a}$ at each of these points is zero, i.e., the singularities are in fact removable. As a consequence, $f_{a}$ can be uniquely extended onto the whole complex plane as a periodic entire function with period $2 \pi \mathrm{i}$. Moreover, since $f_{a}(w)$ vanishes as $\operatorname{Re}(w) \rightarrow \infty$, the function $f_{a}$ is clearly bounded. By invoking Liouville's theorem, we conclude that $f_{a}(w)=0$ for all $w \in \mathbb{C}$, and so

$$
\frac{(L C L)_{a, a}}{F_{a} \bar{F}_{a}}=f_{a}(0)=0
$$


Next, let $a, b \in \mathbb{N}_{n}$ be arbitrary indices satisfying $a \neq b$. Keeping in mind the definitions (3.5) and (3.6), we find at once that

$$
\begin{aligned}
\frac{(L C L)_{a, b}}{F_{a} \bar{F}_{b}}= & \frac{\mathrm{i} \sin (\mu)\left(\mathrm{i} \sin (\mu) z_{a}+\mathrm{i} \sin (\mu-\nu)\right)}{\sinh \left(\mathrm{i} \mu+\lambda_{a}-\lambda_{b}\right) \sinh \left(\mathrm{i} \mu+2 \lambda_{a}\right)}+\frac{\mathrm{i} \sin (\mu)\left(\mathrm{i} \sin (\mu) \bar{z}_{b}+\mathrm{i} \sin (\mu-\nu)\right)}{\sinh \left(\mathrm{i} \mu+\lambda_{a}-\lambda_{b}\right) \sinh \left(\mathrm{i} \mu-2 \lambda_{b}\right)} \\
& +\frac{\mathrm{i} \sin (\mu) \bar{z}_{a}}{\sinh \left(\mathrm{i} \mu-\lambda_{a}-\lambda_{b}\right)}+\frac{\mathrm{i} \sin (\mu) z_{b}}{\sinh \left(\mathrm{i} \mu+\lambda_{a}+\lambda_{b}\right)} \\
& -\sum_{\substack{j=1 \\
(j \neq a, b, n+a, n+b)}}^{N} \frac{\sin (\mu)^{2} z_{j}}{\sinh \left(\mathrm{i} \mu+\lambda_{a}+\Lambda_{j}\right) \sinh \left(\mathrm{i} \mu-\lambda_{b}+\Lambda_{j}\right)} .
\end{aligned}
$$

Although this equation looks considerably more complicated than (3.11), it can be analyzed by the same techniques. Indeed, by replacing $\lambda_{a}$ with $\lambda_{a}+w$ in the right-hand side of (3.14), we may obtain a meromorphic function $f_{a, b}$ of $w \in \mathbb{C}$ that has at most first order poles at the points

$$
w \equiv-\lambda_{a}, w \equiv-\mathrm{i} \mu / 2-\lambda_{a}, w \equiv-\mathrm{i} \mu-\lambda_{a}+\lambda_{b}, w \equiv \Lambda_{j}-\lambda_{a}\left(j \in \mathbb{N}_{N}\right) \quad(\bmod \mathrm{i} \pi) .
$$

However, the residue of $f_{a, b}$ at each of these points turns out to be zero, and $f_{a, b}(w)$ also vanishes as $\operatorname{Re}(w) \rightarrow \infty$. Due to Liouville's theorem we get $f_{a, b}(w)=0$ for all $w \in \mathbb{C}$, thus

$$
\frac{(L C L)_{a, b}}{F_{a} \bar{F}_{b}}=f_{a, b}(0)=0 .
$$

Finally, by taking an arbitrary $a \in \mathbb{N}_{n}$, from (3.5) and (3.6) we see that

$$
(L C L)_{a, n+a}=u_{a}^{2}+\frac{\left(\mathrm{i} \sin (\mu) z_{a}+\mathrm{i} \sin (\mu-\nu)\right)^{2}}{\sinh \left(\mathrm{i} \mu+2 \lambda_{a}\right)^{2}}-\sum_{\substack{j=1 \\ j \neq a, n+a)}}^{N} \frac{\sin (\mu)^{2} z_{a} z_{j}}{\sinh \left(\mathrm{i} \mu+\lambda_{a}+\Lambda_{j}\right)^{2}} .
$$

By replacing $\lambda_{a}$ with $\lambda_{a}+w$ in the right-hand side of (3.17), we end up with a meromorphic function $f_{n+a}$ of the complex variable $w$ that has at most second order poles at the points

$$
w \equiv-\lambda_{a}, w \equiv-\mathrm{i} \mu / 2-\lambda_{a}, w \equiv \Lambda_{j}-\lambda_{a}\left(j \in \mathbb{N}_{N}\right) \quad(\bmod \mathrm{i} \pi) .
$$

Though the calculations are a bit more involved as in the previous cases, one can show that the singularities of $f_{n+a}$ are actually removable. Moreover, it is evident that $f_{n+a}(w) \rightarrow 1$ as $\operatorname{Re}(w) \rightarrow \infty$. Liouville's theorem applies again, implying that $f_{n+a}(w)=1$ for all $w \in \mathbb{C}$. Thus the relationship

$$
(L C L)_{a, n+a}=f_{n+a}(0)=1
$$

also follows, whence the proof is complete.

In the earlier paper [10] we saw that the rational analogue of $L(3.6)$ takes values in the symmetric space $\exp (\mathfrak{p})$ (2.9). We find it reassuring that the proof of Lemma 7 of paper [10] allows a straightforward generalization into the present hyperbolic context, too.

Lemma 2. At each point of the phase space we have $L \in \exp (\mathfrak{p})$. 
Proof. Recalling the identification (2.8) and Proposition 1, it is enough to prove that the Hermitian matrix $L(3.6)$ is positive definite. For this reason, take an arbitrary point $(\lambda, \theta) \in P$ and keep it fixed. To prove the Lemma, below we offer a standard continuity argument by analyzing the dependence of $L$ solely on the coupling parameters.

In the very special case when the pair $(\mu, \nu)$ formed by the coupling parameters obey the relationship $\sin (\mu-\nu)=0$, the Lax matrix $L$ (3.6) becomes a hyperbolic Cauchy-like matrix and the generalized Cauchy determinant formula (see e.g. equation (1.2) in [17]) readily implies the positivity of all its leading principal minors. Thus, recalling Sylvester's criterion, we conclude that $L$ is positive definite.

Turning to the general case, suppose that the pair $(\mu, \nu)$ is restricted only by the conditions displayed in (1.6). It is clear that in the 2-dimensional space of the admissible coupling parameters characterized by (1.6) one can find a continuous curve with endpoints $(\mu, \nu)$ and $\left(\mu_{0}, \nu_{0}\right)$, where $\mu_{0}$ and $\nu_{0}$ satisfy the additional requirement $\sin \left(\mu_{0}-\nu_{0}\right)=0$. Since the dependence of the Hermitian matrix $L$ on the coupling parameters is smooth, along this curve the smallest eigenvalue of $L$ moves continuously. However, it cannot cross zero, since by Proposition 1 the matrix $L$ remains invertible during this deformation. Therefore, since the eigenvalues of $L$ are strictly positive at the endpoint $\left(\mu_{0}, \nu_{0}\right)$, they must be strictly positive at the other endpoint $(\mu, \nu)$ as well.

\subsection{Commutation relation and regularity}

As Ruijsenaars has observed in his seminal paper on the translation invariant CMS and RS type pure soliton systems, one of the key ingredients in their analysis is the fact that their Lax matrices obey certain non-trivial commutation relations with some diagonal matrices (for details, see equation (2.4) and the surrounding ideas in [17]). As a momentum map constraint, an analogous commutation relation has also played a key role in the geometric study of the rational $C_{n}$ and $B C_{n}$ RSvD systems (see [10, 11, 13]). Due to its importance, our first goal in this subsection is to set up a Ruijsenaars type commutation relation for the proposed Lax matrix $L$ (3.6), too. As a technical remark, we mention in passing that from now on we shall apply frequently the standard functional calculus on the linear operators $\operatorname{ad}_{\boldsymbol{\Lambda}}(2.16)$ and $\widetilde{a d}_{\boldsymbol{\Lambda}}$ (2.17) associated with the diagonal matrix $\Lambda \in \mathfrak{c}($ (3.2).

Lemma 3. The matrix $L(3.6)$ and the diagonal matrix $e^{\boldsymbol{\Lambda}}$ obey the Ruijsenaars type commutation relation

$$
e^{\mathrm{i} \mu} e^{\operatorname{ad}_{\Lambda}} L-e^{-\mathrm{i} \mu} e^{-\mathrm{ad}_{\Lambda}} L=2 \mathrm{i} \sin (\mu) F F^{*}+2 \mathrm{i} \sin (\mu-\nu) C .
$$

Proof. Recalling the matrix entries of $L$, for all $k, l \in \mathbb{N}_{N}$ we can write that

$$
\begin{aligned}
& \left(e^{\mathrm{i} \mu} e^{\mathrm{ad}_{\Lambda}} L-e^{-\mathrm{i} \mu} e^{-\mathrm{ad}_{\Lambda}} L\right)_{k, l}=\left(e^{\mathrm{i} \mu} e^{\boldsymbol{\Lambda}} L e^{-\boldsymbol{\Lambda}}-e^{-\mathrm{i} \mu} e^{-\boldsymbol{\Lambda}} L e^{\boldsymbol{\Lambda}}\right)_{k, l} \\
& =e^{\mathrm{i} \mu} e^{\Lambda_{k}} L_{k, l} e^{-\Lambda_{l}}-e^{-\mathrm{i} \mu} e^{-\Lambda_{k}} L_{k, l} e^{\Lambda_{l}}=2 \sinh \left(\mathrm{i} \mu+\Lambda_{k}-\Lambda_{l}\right) L_{k, l} \\
& =2 \mathrm{i} \sin (\mu) F_{k} \bar{F}_{l}+2 \mathrm{i} \sin (\mu-\nu) C_{k, l}=\left(2 \mathrm{i} \sin (\mu) F F^{*}+2 \mathrm{i} \sin (\mu-\nu) C\right)_{k, l},
\end{aligned}
$$

thus (3.20) follows at once.

Though the proof of Lemma 3 is almost trivial, it proves to be quite handy in the forthcoming calculations. In particular, based on the commutation relation (3.20), we shall now prove that 
the spectrum of $L$ is simple. Heading toward our present goal, first let us recall that Lemma 2 tells us that $L \in \exp (\mathfrak{p})$. Therefore, as we can infer easily from (2.11), one can find some $y \in K$ and a real $n$-tuple $\hat{\theta}=\left(\hat{\theta}_{1}, \ldots, \hat{\theta}_{n}\right) \in \mathbb{R}^{n}$ satisfying

$$
\hat{\theta}_{1} \geq \ldots \geq \hat{\theta}_{n} \geq 0
$$

such that with the diagonal matrix

$$
\hat{\boldsymbol{\Theta}}=\operatorname{diag}\left(\hat{\Theta}_{1}, \ldots, \hat{\Theta}_{N}\right)=\operatorname{diag}\left(\hat{\theta}_{1}, \ldots, \hat{\theta}_{n},-\hat{\theta}_{1}, \ldots,-\hat{\theta}_{n}\right) \in \mathfrak{a}
$$

we can write

$$
L=y e^{2 \hat{\mathbf{\Theta}}} y^{-1} .
$$

Now, upon defining

$$
\hat{L}=y^{-1} e^{2 \boldsymbol{\Lambda}} y \in \exp (\mathfrak{p}) \text { and } \hat{F}=e^{-\hat{\boldsymbol{\Theta}}} y^{-1} e^{\boldsymbol{\Lambda}} F \in \mathbb{C}^{N},
$$

for these new objects we can also set up a commutation relation analogous to (3.20). Indeed, from (3.20) one can derive that

$$
e^{\mathrm{i} \mu} e^{-\hat{\boldsymbol{\Theta}}} \hat{L} e^{\hat{\boldsymbol{\Theta}}}-e^{-\mathrm{i} \mu} e^{\hat{\boldsymbol{\Theta}}} \hat{L} e^{-\hat{\boldsymbol{\Theta}}}=2 \mathrm{i} \sin (\mu) \hat{F} \hat{F}^{*}+2 \mathrm{i} \sin (\mu-\nu) C .
$$

Componentwise, from (3.26) we conclude that

$$
\hat{L}_{k, l}=\frac{\mathrm{i} \sin (\mu) \hat{F}_{k} \overline{\hat{F}}_{l}+\mathrm{i} \sin (\mu-\nu) C_{k, l}}{\sinh \left(\mathrm{i} \mu-\hat{\Theta}_{k}+\hat{\Theta}_{l}\right)} \quad\left(k, l \in \mathbb{N}_{N}\right) .
$$

Since $\hat{L}(\underline{3.25})$ is a positive definite matrix, its diagonal entries are strictly positive. Therefore, by exploiting (3.27), we can write

$$
0<\hat{L}_{k, k}=\left|\hat{F}_{k}\right|^{2} \text {. }
$$

The upshot of this trivial observation is that $\hat{F}_{k} \neq 0$ for all $k \in \mathbb{N}_{N}$.

To proceed further, notice that for the inverse matrix $\hat{L}^{-1}=C \hat{L} C$ we can also cook up an equation analogous to (3.26). Indeed, by simply multiplying both sides of (3.26) with the matrix $C(2.2)$, we obtain

$$
e^{\mathrm{i} \mu} e^{\hat{\Theta}} \hat{L}^{-1} e^{-\hat{\Theta}}-e^{-\mathrm{i} \mu} e^{-\hat{\Theta}} \hat{L}^{-1} e^{\hat{\Theta}}=2 \mathrm{i} \sin (\mu)(C \hat{F})(C \hat{F})^{*}+2 \mathrm{i} \sin (\mu-\nu) C,
$$

that leads immediately to the matrix entries

$$
\left(\hat{L}^{-1}\right)_{k, l}=\frac{\mathrm{i} \sin (\mu)(C \hat{F})_{k} \overline{(C \hat{F})_{l}}+\mathrm{i} \sin (\mu-\nu) C_{k, l}}{\sinh \left(\mathrm{i} \mu+\hat{\Theta}_{k}-\hat{\Theta}_{l}\right)} \quad\left(k, l \in \mathbb{N}_{N}\right) .
$$

For further reference, we now spell out the trivial equation

$$
\delta_{k, l}=\sum_{j=1}^{N} \hat{L}_{k, j}\left(\hat{L}^{-1}\right)_{j, l}
$$


for certain values of $k, l \in \mathbb{N}_{N}$. First, by plugging the explicit formulae (3.27) and (3.30) into the relationship (3.31), with the special choice of indices $k=l=a \in \mathbb{N}_{n}$ one finds that

$$
\begin{aligned}
0=1 & +\frac{\sin (\mu-\nu)^{2}}{\sinh \left(\mathrm{i} \mu-2 \hat{\theta}_{a}\right)^{2}}+\frac{2 \sin (\mu) \sin (\mu-\nu) \hat{F}_{a} \overline{\hat{F}}_{n+a}}{\sinh \left(\mathrm{i} \mu-2 \hat{\theta}_{a}\right)^{2}} \\
& +\sin (\mu)^{2} \hat{F}_{a} \overline{\hat{F}}_{n+a} \sum_{c=1}^{n}\left(\frac{\hat{\hat{F}}_{c} \hat{F}_{n+c}}{\sinh \left(\mathrm{i} \mu-\hat{\theta}_{a}+\hat{\theta}_{c}\right)^{2}}+\frac{\hat{F}_{c} \overline{\hat{F}}_{n+c}}{\sinh \left(\mathrm{i} \mu-\hat{\theta}_{a}-\hat{\theta}_{c}\right)^{2}}\right) .
\end{aligned}
$$

Second, if $k=a$ and $l=n+a$ with some $a \in \mathbb{N}_{n}$, then from (3.31) we obtain

$$
\begin{aligned}
& \sin (\mu)^{2} \sum_{c=1}^{n}\left(\frac{\overline{\hat{F}}_{c} \hat{F}_{n+c}}{\sinh \left(\mathrm{i} \mu-\hat{\theta}_{a}+\hat{\theta}_{c}\right) \sinh \left(\mathrm{i} \mu+\hat{\theta}_{c}+\hat{\theta}_{a}\right)}+\frac{\hat{F}_{c} \overline{\hat{F}}_{n+c}}{\sinh \left(\mathrm{i} \mu-\hat{\theta}_{a}-\hat{\theta}_{c}\right) \sinh \left(\mathrm{i} \mu-\hat{\theta}_{c}+\hat{\theta}_{a}\right)}\right) \\
& \quad=\mathrm{i} \sin (\mu-\nu)\left(\frac{1}{\sinh \left(\mathrm{i} \mu-2 \hat{\theta}_{a}\right)}+\frac{1}{\sinh \left(\mathrm{i} \mu+2 \hat{\theta}_{a}\right)}\right) .
\end{aligned}
$$

Third, if $k=a$ and $l=b$ with some $a, b \in \mathbb{N}_{n}$ satisfying $a \neq b$, then the relationship (3.31) immediately leads to the equation

$$
\begin{aligned}
& \sin (\mu)^{2} \sum_{c=1}^{n}\left(\frac{\overline{\hat{F}}_{c} \hat{F}_{n+c}}{\sinh \left(\mathrm{i} \mu-\hat{\theta}_{a}+\hat{\theta}_{c}\right) \sinh \left(\mathrm{i} \mu+\hat{\theta}_{c}-\hat{\theta}_{b}\right)}+\frac{\hat{F}_{c} \overline{\hat{F}}_{n+c}}{\sinh \left(\mathrm{i} \mu-\hat{\theta}_{a}-\hat{\theta}_{c}\right) \sinh \left(\mathrm{i} \mu-\hat{\theta}_{c}-\hat{\theta}_{b}\right)}\right) \\
& =-\frac{\sin (\mu) \sin (\mu-\nu)}{\sinh \left(\mathrm{i} \mu-\hat{\theta}_{a}-\hat{\theta}_{b}\right)}\left(\frac{1}{\sinh \left(\mathrm{i} \mu-2 \hat{\theta}_{a}\right)}+\frac{1}{\sinh \left(\mathrm{i} \mu-2 \hat{\theta}_{b}\right)}\right) .
\end{aligned}
$$

At this point we wish to emphasize that during the derivation of the last two equations (3.33) and (3.34) it proves to be essential that each component of the column vector $\hat{F}$ (3.25) is nonzero, as we have seen in (3.28).

Lemma 4. Under the additional assumption on the coupling parameters

$$
\sin (2 \mu-\nu) \neq 0
$$

the spectrum of the matrix $L(3.6)$ is simple of the form

$$
\operatorname{Spec}(L)=\left\{e^{ \pm 2 \hat{\theta}_{a}} \mid a \in \mathbb{N}_{n}\right\},
$$

where $\hat{\theta}_{1}>\ldots>\hat{\theta}_{n}>0$. In other words, $L$ is regular in the sense that $L \in \exp \left(\mathfrak{p}_{\mathrm{reg}}\right)$.

Proof. First, let us suppose that $\hat{\theta}_{a}=0$ for some $a \in \mathbb{N}_{n}$. With this particular index $a$, from equation (3.32) we infer that

$$
\begin{aligned}
0=1 & -\frac{\sin (\mu-\nu)^{2}}{\sin (\mu)^{2}}-\frac{2 \sin (\mu-\nu) \hat{F}_{a} \overline{\hat{F}}_{n+a}}{\sin (\mu)} \\
& +\sin (\mu)^{2} \hat{F}_{a} \overline{\hat{F}}_{n+a} \sum_{c=1}^{n}\left(\frac{\overline{\hat{F}}_{c} \hat{F}_{n+c}}{\sinh \left(\mathrm{i} \mu+\hat{\theta}_{c}\right)^{2}}+\frac{\hat{F}_{c} \overline{\hat{F}}_{n+c}}{\sinh \left(\mathrm{i} \mu-\hat{\theta}_{c}\right)^{2}}\right),
\end{aligned}
$$


while (3.33) leads to the relationship

$$
\sin (\mu)^{2} \sum_{c=1}^{n}\left(\frac{\overline{\hat{F}}_{c} \hat{F}_{n+c}}{\sinh \left(\mathrm{i} \mu+\hat{\theta}_{c}\right)^{2}}+\frac{\hat{F}_{c} \overline{\hat{F}}_{n+c}}{\sinh \left(\mathrm{i} \mu-\hat{\theta}_{c}\right)^{2}}\right)=\frac{2 \sin (\mu-\nu)}{\sin (\mu)} .
$$

Now, by plugging (3.38) into (3.37), we obtain

$$
0=1-\frac{\sin (\mu-\nu)^{2}}{\sin (\mu)^{2}}=\frac{\sin (\mu)^{2}-\sin (\mu-\nu)^{2}}{\sin (\mu)^{2}}=\frac{\sin (\nu) \sin (2 \mu-\nu)}{\sin (\mu)^{2}},
$$

which clearly contradicts the assumptions imposed in the equations (1.6) and (3.35). Thus, we are forced to conclude that for all $a \in \mathbb{N}_{n}$ we have $\hat{\theta}_{a} \neq 0$.

Second, let us suppose that $\hat{\theta}_{a}=\hat{\theta}_{b}$ for some $a, b \in \mathbb{N}_{n}$ satisfying $a \neq b$. With these particular indices $a$ and $b$, equation (3.34) takes the form

$$
\sin (\mu)^{2} \sum_{c=1}^{n}\left(\frac{\overline{\hat{F}}_{c} \hat{F}_{n+c}}{\sinh \left(\mathrm{i} \mu-\hat{\theta}_{a}+\hat{\theta}_{c}\right)^{2}}+\frac{\hat{F}_{c} \overline{\hat{F}}_{n+c}}{\sinh \left(\mathrm{i} \mu-\hat{\theta}_{a}-\hat{\theta}_{c}\right)^{2}}\right)=-\frac{2 \sin (\mu) \sin (\mu-\nu)}{\sinh \left(\mathrm{i} \mu-2 \hat{\theta}_{a}\right)^{2}}
$$

Now, by plugging this formula into (3.32), we obtain immediately that

$$
0=1+\frac{\sin (\mu-\nu)^{2}}{\sinh \left(\mathrm{i} \mu-2 \hat{\theta}_{a}\right)^{2}},
$$

which in turn implies that

$$
\begin{aligned}
& \sin (\mu-\nu)^{2}=-\sinh \left(\mathrm{i} \mu-2 \hat{\theta}_{a}\right)^{2} \\
& \quad=\sin (\mu)^{2} \cosh \left(2 \hat{\theta}_{a}\right)^{2}-\cos (\mu)^{2} \sinh \left(2 \hat{\theta}_{a}\right)^{2}+\mathrm{i} \sin (\mu) \cos (\mu) \sinh \left(4 \hat{\theta}_{a}\right) .
\end{aligned}
$$

Since $\hat{\theta}_{a} \neq 0$ and since $\sin (\mu) \neq 0$, the imaginary part of the above equation leads to the relation $\cos (\mu)=0$, whence $\sin (\mu)^{2}=1$ also follows. Now, by plugging these observations into the real part of (3.42), we end up with the contradiction

$$
1 \geq \sin (\mu-\nu)^{2}=\cosh \left(2 \hat{\theta}_{a}\right)^{2}>1 .
$$

Thus, if $a, b \in \mathbb{N}_{n}$ and $a \neq b$, then necessarily we have $\hat{\theta}_{a} \neq \hat{\theta}_{b}$.

Since the spectrum of $L(3.6)$ is simple, it follows that the dependence of the eigenvalues on the matrix entries is smooth. Therefore, recalling (3.36), it is clear that each $\hat{\theta}_{c}\left(c \in \mathbb{N}_{n}\right)$ can be seen as a smooth function on $P(1.2)$, i.e.,

$$
\hat{\theta}_{c} \in C^{\infty}(P) .
$$

To conclude this subsection, we also offer a few remarks on the additional constraint appearing in (3.35), that we keep in effect in the rest of the paper. Naively, this assumption excludes a 1-dimensional subset from the 2-dimensional space of the parameters $(\mu, \nu)$. However, looking back to the Hamiltonian $H(\underline{1.5})$, it is clear that the effective coupling constants of our van 
Diejen systems are rather the positive numbers $\sin (\mu)^{2}$ and $\sin (\nu)^{2}$. Therefore, keeping in mind (1.6), on the parameters $\mu$ and $\nu$ we could have imposed the requirement, say,

$$
(\mu, \nu) \in((0, \pi / 4) \times[-\pi / 2,0)) \cup([\pi / 4, \pi / 2] \times(0, \pi / 2]),
$$

at the outset. The point is that, under the requirement (3.45), the equation $\sin (2 \mu-\nu)=0$ is equivalent to the pair of equations $\sin (\mu)^{2}=1 / 2$ and $\sin (\nu)^{2}=1$. To put it differently, our observation is that under the assumptions (1.6) and (3.35) the pair $\left(\sin (\mu)^{2}, \sin (\nu)^{2}\right)$ formed by the relevant coupling constants can take on any values from the 'square' $(0,1] \times(0,1]$, except the single point $(1 / 2,1)$. From the proof of Lemma 4, especially from equation (3.38), one may get the impression that even this very slight technical assumption can be relaxed by further analyzing the properties of column vector $\hat{F}(3.25)$. However, we do not wish to pursue this direction in the present paper.

\section{Analyzing the dynamics}

In this section we wish to study the dynamics generated by the Hamiltonian $H$ (1.5). Recalling the formulae (3.4) and (3.6), by the obvious relationship

$$
H=\sum_{c=1}^{n} \cosh \left(\theta_{c}\right) u_{c}=\frac{1}{2} \operatorname{tr}(L)
$$

we can make the first contact of our van Diejen system with the proposed Lax matrix $L$ (3.6) . As an important ingredient of the forthcoming analysis, let us introduce the Hamiltonian vector field $\boldsymbol{X}_{H} \in \mathfrak{X}(P)$ with the usual definition

$$
\boldsymbol{X}_{H}[f]=\{f, H\} \quad\left(f \in C^{\infty}(P)\right) .
$$

Working with the convention (1.4), for the time evolution of the global coordinate functions $\lambda_{a}$ and $\theta_{a}\left(a \in \mathbb{N}_{n}\right)$ we can clearly write

$$
\begin{aligned}
& \dot{\lambda}_{a}=\boldsymbol{X}_{H}\left[\lambda_{a}\right]=\frac{\partial H}{\partial \theta_{a}}=\sinh \left(\theta_{a}\right) u_{a} \\
& \dot{\theta}_{a}=\boldsymbol{X}_{H}\left[\theta_{a}\right]=-\frac{\partial H}{\partial \lambda_{a}}=-\sum_{c=1}^{n} \cosh \left(\theta_{c}\right) u_{c} \frac{\partial \ln \left(u_{c}\right)}{\partial \lambda_{a}} .
\end{aligned}
$$

To make the right-hand side of (4.4) more explicit, let us display the logarithmic derivatives of the constituent functions $u_{c}$. Notice that for all $a \in \mathbb{N}_{n}$ we can write

$$
\frac{\partial \ln \left(u_{a}\right)}{\partial \lambda_{a}}=-\operatorname{Re}\left(\frac{2 \mathrm{i} \sin (\nu)}{\sinh \left(2 \lambda_{a}\right) \sinh \left(\mathrm{i} \nu+2 \lambda_{a}\right)}+\sum_{\substack{j=1 \\ j \neq a, n+a)}}^{N} \frac{\mathrm{i} \sin (\mu)}{\sinh \left(\lambda_{a}-\Lambda_{j}\right) \sinh \left(\mathrm{i} \mu+\lambda_{a}-\Lambda_{j}\right)}\right),
$$

while if $c \in \mathbb{N}_{n}$ and $c \neq a$, then we have

$$
\frac{\partial \ln \left(u_{c}\right)}{\partial \lambda_{a}}=\operatorname{Re}\left(\frac{\mathrm{i} \sin (\mu)}{\sinh \left(\lambda_{a}-\lambda_{c}\right) \sinh \left(\mathrm{i} \mu+\lambda_{a}-\lambda_{c}\right)}-\frac{\mathrm{i} \sin (\mu)}{\sinh \left(\lambda_{a}+\lambda_{c}\right) \sinh \left(\mathrm{i} \mu+\lambda_{a}+\lambda_{c}\right)}\right) .
$$

The rest of this section is devoted to the study of the Hamiltonian dynamical system (4.3)-(4.4). 


\subsection{Completeness of the Hamiltonian vector field}

Undoubtedly, the Hamiltonian (1.5) does not take the usual form one finds in the standard textbooks on classical mechanics. It is thus inevitable that we have even less intuition about the generated dynamics than in the case of the 'natural systems' characterized by a kinetic term plus a potential. To get a finer picture about the solutions of the Hamiltonian dynamics (4.3)-(4.4), we start our study with a brief analysis on the completeness of the Hamiltonian vector field $\boldsymbol{X}_{H}$ (4.2).

As the first step, we introduce the strictly positive constant

$$
\mathcal{S}=\min \{|\sin (\mu)|,|\sin (\nu)|\} \in(0,1]
$$

Giving a glance at (3.4), it is evident that

$$
u_{n}>\left(1+\frac{\sin (\nu)^{2}}{\sinh \left(2 \lambda_{n}\right)^{2}}\right)^{\frac{1}{2}}>\frac{|\sin (\nu)|}{\sinh \left(2 \lambda_{n}\right)} \geq \frac{\mathcal{S}}{\sinh \left(2 \lambda_{n}\right)},
$$

while for all $c \in \mathbb{N}_{n-1}$ we can write

$$
u_{c}>\left(1+\frac{\sin (\mu)^{2}}{\sinh \left(\lambda_{c}-\lambda_{c+1}\right)^{2}}\right)^{\frac{1}{2}}>\frac{|\sin (\mu)|}{\sinh \left(\lambda_{c}-\lambda_{c+1}\right)} \geq \frac{\mathcal{S}}{\sinh \left(\lambda_{c}-\lambda_{c+1}\right)} .
$$

Keeping in mind the above trivial inequalities, we are ready to prove the following result.

Theorem 5. The Hamiltonian vector field $\boldsymbol{X}_{H}(4.2)$ generated by the van Diejen type Hamiltonian function $H$ (1.5) is complete. That is, the maximum interval of existence of each integral curve of $\boldsymbol{X}_{H}$ is the whole real axis $\mathbb{R}$.

Proof. Take an arbitrary point

$$
\gamma_{0}=\left(\lambda^{(0)}, \theta^{(0)}\right) \in P
$$

and let

$$
\gamma:(\alpha, \beta) \rightarrow P, \quad t \mapsto \gamma(t)=(\lambda(t), \theta(t))
$$

be the unique maximally defined integral curve of $\boldsymbol{X}_{H}$ with $-\infty \leq \alpha<0<\beta \leq \infty$ satisfying the initial condition $\gamma(0)=\gamma_{0}$. Since the Hamiltonian $H$ is smooth, the existence, the uniqueness, and also the smoothness of such a maximal solution are obvious. Our goal is to show that for the domain of the maximally defined trajectory $\gamma$ (4.11) we have $(\alpha, \beta)=\mathbb{R}$; that is, $\alpha=-\infty$ and $\beta=\infty$.

Arguing by contradiction, first let us suppose that $\beta<\infty$. Since the Hamiltonian $H$ is a first integral, for all $t \in(\alpha, \beta)$ and for all $a \in \mathbb{N}_{n}$ we can write

$$
H\left(\gamma_{0}\right)=H(\gamma(t))=\sum_{c=1}^{n} \cosh \left(\theta_{c}(t)\right) u_{c}(\lambda(t))>\cosh \left(\theta_{a}(t)\right) u_{a}(\lambda(t))
$$

whence the estimation

$$
H\left(\gamma_{0}\right)>\cosh \left(\left|\theta_{a}(t)\right|\right) \geq \frac{1}{2} e^{\left|\theta_{a}(t)\right|}
$$


is also immediate. Thus, upon introducing the cube

$$
\mathcal{C}=\left[-\ln \left(2 H\left(\gamma_{0}\right)\right), \ln \left(2 H\left(\gamma_{0}\right)\right)\right]^{n} \subseteq \mathbb{R}^{n}
$$

from (4.13) we infer at once that

$$
\theta(t) \in \mathcal{C} \quad(t \in(\alpha, \beta)) .
$$

Turning to the equations (4.3) and (4.12), we can cook up an estimation on the growing of the vector $\lambda(t)$, too. Indeed, we see that

$$
\left|\dot{\lambda}_{1}(t)\right|=\sinh \left(\left|\theta_{1}(t)\right|\right) u_{1}(\lambda(t)) \leq \cosh \left(\left|\theta_{1}(t)\right|\right) u_{1}(\lambda(t))<H\left(\gamma_{0}\right) \quad(t \in(\alpha, \beta)),
$$

that implies immediately that for all $t \in[0, \beta)$ we have

$$
\left|\lambda_{1}(t)-\lambda_{1}^{(0)}\right|=\left|\lambda_{1}(t)-\lambda_{1}(0)\right|=\left|\int_{0}^{t} \dot{\lambda}_{1}(s) \mathrm{d} s\right| \leq \int_{0}^{t}\left|\dot{\lambda}_{1}(s)\right| \mathrm{d} s \leq t H\left(\gamma_{0}\right)<\beta H\left(\gamma_{0}\right) .
$$

Therefore, with the aid of the strictly positive constant

$$
\rho=\lambda_{1}^{(0)}+\beta H\left(\gamma_{0}\right) \in(0, \infty)
$$

we end up with the estimation

$$
\lambda_{1}(t)=\left|\lambda_{1}(t)\right|=\left|\lambda_{1}^{(0)}+\lambda_{1}(t)-\lambda_{1}^{(0)}\right| \leq\left|\lambda_{1}^{(0)}\right|+\left|\lambda_{1}(t)-\lambda_{1}^{(0)}\right|<\rho \quad(t \in[0, \beta)) .
$$

Since $\lambda(t)$ moves in the configuration space $Q$ (1.1), the above observation entails that

$$
\rho>\lambda_{1}(t)>\ldots>\lambda_{n}(t)>0 \quad(t \in[0, \beta)) .
$$

To proceed further, now for all $\varepsilon>0$ we define the subset $Q_{\varepsilon} \subseteq \mathbb{R}^{n}$ consisting of those real $n$-tuples $x=\left(x_{1}, \ldots, x_{n}\right) \in \mathbb{R}^{n}$ that satisfy the inequalities

$$
\rho \geq x_{1} \text { and } 2 x_{n} \geq \varepsilon \text { and } x_{c} \geq x_{c+1}+\varepsilon \text { for all } c \in \mathbb{N}_{n-1},
$$

simultaneously. In other words,

$$
Q_{\varepsilon}=\left\{x \in \mathbb{R}^{n} \mid \rho \geq x_{1}\right\} \cap\left\{x \in \mathbb{R}^{n} \mid 2 x_{n} \geq \varepsilon\right\} \cap \bigcap_{c=1}^{n-1}\left\{x \in \mathbb{R}^{n} \mid x_{c}-x_{c+1} \geq \varepsilon\right\} .
$$

Notice that $Q_{\varepsilon}$ is a bounded and closed subset of $\mathbb{R}^{n}$. Moreover, by comparing the definitions (1.1) and (4.21), it is evident that $Q_{\varepsilon} \subseteq Q$. Since the cube $\mathcal{C}$ (4.14) is also a compact subset of $\mathbb{R}^{n}$, we conclude that the Cartesian product $Q_{\varepsilon} \times \mathcal{C}$ is a compact subset of the phase space $P$ (1.2). Therefore, due to the assumption $\beta<\infty$, after some time the maximally defined trajectory $\gamma$ (4.11) escapes from $Q_{\varepsilon} \times \mathcal{C}$, as can be read off from any standard reference on dynamical systems (see e.g. Theorem 2.1.18 in [18]). More precisely, there is some $\tau_{\varepsilon} \in[0, \beta$ ) such that

$$
(\lambda(t), \theta(t)) \in P \backslash\left(Q_{\varepsilon} \times \mathcal{C}\right)=\left(\left(Q \backslash Q_{\varepsilon}\right) \times \mathcal{C}\right) \cup\left(Q \times\left(\mathbb{R}^{n} \backslash \mathcal{C}\right)\right) \quad\left(t \in\left(\tau_{\varepsilon}, \beta\right)\right),
$$


where the union above is actually a disjoint union. For instance, due to the relationship (4.15), at the mid-point

$$
t_{\varepsilon}=\frac{\tau_{\varepsilon}+\beta}{2} \in\left(\tau_{\varepsilon}, \beta\right)
$$

we can write that

$$
\lambda\left(t_{\varepsilon}\right) \in Q \backslash Q_{\varepsilon} \subseteq \mathbb{R}^{n} \backslash Q_{\varepsilon} .
$$

Therefore, simply by taking the complement of $Q_{\varepsilon}$ (4.22), and also keeping in mind (4.19), it is evident that

$$
\min \left\{\lambda_{1}\left(t_{\varepsilon}\right)-\lambda_{2}\left(t_{\varepsilon}\right), \ldots, \lambda_{n-1}\left(t_{\varepsilon}\right)-\lambda_{n}\left(t_{\varepsilon}\right), 2 \lambda_{n}\left(t_{\varepsilon}\right)\right\}<\varepsilon
$$

which in turn implies that

$$
\max \left\{\frac{1}{\sinh \left(\lambda_{1}\left(t_{\varepsilon}\right)-\lambda_{2}\left(t_{\varepsilon}\right)\right)}, \ldots, \frac{1}{\sinh \left(\lambda_{n-1}\left(t_{\varepsilon}\right)-\lambda_{n}\left(t_{\varepsilon}\right)\right)}, \frac{1}{\sinh \left(2 \lambda_{n}\left(t_{\varepsilon}\right)\right)}\right\}>\frac{1}{\sinh (\varepsilon)} .
$$

Now, since $\varepsilon>0$ was arbitrary, the estimations (4.8) and (4.9) immediately lead to the contradiction

$$
\begin{aligned}
H\left(\gamma_{0}\right) & =H\left(\gamma\left(t_{\varepsilon}\right)\right)=\sum_{c=1}^{n} \cosh \left(\theta_{c}\left(t_{\varepsilon}\right)\right) u_{c}\left(\lambda\left(t_{\varepsilon}\right)\right) \\
& \geq \sum_{c=1}^{n} u_{c}\left(\lambda\left(t_{\varepsilon}\right)\right)>\frac{\mathcal{S}}{\sinh \left(2 \lambda_{n}\left(t_{\varepsilon}\right)\right)}+\sum_{c=1}^{n-1} \frac{\mathcal{S}}{\sinh \left(\lambda_{c}\left(t_{\varepsilon}\right)-\lambda_{c+1}\left(t_{\varepsilon}\right)\right)}>\frac{\mathcal{S}}{\sinh (\varepsilon)} .
\end{aligned}
$$

Therefore, necessarily, $\beta=\infty$.

Either by repeating the above ideas, or by invoking a time-reversal argument, one can also show that $\alpha=-\infty$, whence the proof is complete.

\subsection{Dynamics of the vector $F$}

Looking back to the definition (3.6), we see that the column vector $F$ (3.5) is important building block of the matrix $L$. Therefore, the study of the derivative of $L$ along the Hamiltonian vector field $\boldsymbol{X}_{H}$ (4.2) does require close control over the derivative of the components of $F$, too. Upon introducing the auxiliary functions

$$
\varphi_{k}=\frac{1}{F_{k}} \boldsymbol{X}_{H}\left[F_{k}\right] \quad\left(k \in \mathbb{N}_{N}\right)
$$

for all $a \in \mathbb{N}_{n}$ we can write

$$
\begin{aligned}
2 \varphi_{a} & =\boldsymbol{X}_{H}\left[\ln \left(F_{a}^{2}\right)\right]=\boldsymbol{X}_{H}\left[\theta_{a}+\ln \left(u_{a}\right)\right]=\left\{\theta_{a}+\ln \left(u_{a}\right), H\right\} \\
& =\sum_{c=1}^{n}\left(\sinh \left(\theta_{c}\right) u_{c} \frac{\partial \ln \left(u_{a}\right)}{\partial \lambda_{c}}-\cosh \left(\theta_{c}\right) u_{c} \frac{\partial \ln \left(u_{c}\right)}{\partial \lambda_{a}}\right) .
\end{aligned}
$$

Therefore, due to the explicit formulae (4.5) and (4.6), we have complete control over the first $n$ components of (4.29). Turning to the remaining components, from the definition (3.5) it is evident that $F_{n+a}=F_{a}^{-1} \bar{z}_{a}$, whence the relationship

$$
\varphi_{n+a}=-\varphi_{a}+\frac{1}{\bar{z}_{a}} \boldsymbol{X}_{H}\left[\bar{z}_{a}\right]=-\varphi_{a}+\sum_{c=1}^{n} \sinh \left(\theta_{c}\right) u_{c} \frac{1}{\bar{z}_{a}} \frac{\partial \bar{z}_{a}}{\partial \lambda_{c}}
$$


follows immediately. Notice that for all $a \in \mathbb{N}_{n}$ we can write that

$$
\frac{1}{z_{a}} \frac{\partial z_{a}}{\partial \lambda_{a}}=-\frac{2 \mathrm{i} \sin (\nu)}{\sinh \left(2 \lambda_{a}\right) \sinh \left(\mathrm{i} \nu+2 \lambda_{a}\right)}-\sum_{\substack{j=1 \\ j \neq a, n+a)}}^{N} \frac{\mathrm{i} \sin (\mu)}{\sinh \left(\lambda_{a}-\Lambda_{j}\right) \sinh \left(\mathrm{i} \mu+\lambda_{a}-\Lambda_{j}\right)},
$$

whereas if $c \in \mathbb{N}_{n}$ and $c \neq a$, then we find immediately that

$$
\frac{1}{z_{a}} \frac{\partial z_{a}}{\partial \lambda_{c}}=\frac{\mathrm{i} \sin (\mu)}{\sinh \left(\lambda_{a}-\lambda_{c}\right) \sinh \left(\mathrm{i} \mu+\lambda_{a}-\lambda_{c}\right)}-\frac{\mathrm{i} \sin (\mu)}{\sinh \left(\lambda_{a}+\lambda_{c}\right) \sinh \left(\mathrm{i} \mu+\lambda_{a}+\lambda_{c}\right)} .
$$

The above observations can be summarized as follows.

Proposition 6. For the derivative of the components of the function F (3.5) along the Hamiltonian vector field $\boldsymbol{X}_{H}$ (4.2) we have

$$
\boldsymbol{X}_{H}\left[F_{k}\right]=\varphi_{k} F_{k} \quad\left(k \in \mathbb{N}_{N}\right)
$$

where for each $a \in \mathbb{N}_{n}$ we can write

$$
\varphi_{a}=\operatorname{Re}\left(\frac{\mathrm{i} \sin (\nu) e^{-\theta_{a}} u_{a}}{\sinh \left(2 \lambda_{a}\right) \sinh \left(\mathrm{i} \nu+2 \lambda_{a}\right)}+\frac{1}{2} \sum_{\substack{j=1 \\ j \neq a, n+a)}}^{N} \frac{\mathrm{i} \sin (\mu)\left(e^{-\theta_{a}} u_{a}+e^{\Theta_{j}} u_{j}\right)}{\sinh \left(\lambda_{a}-\Lambda_{j}\right) \sinh \left(\mathrm{i} \mu+\lambda_{a}-\Lambda_{j}\right)}\right)
$$

whereas

$$
\varphi_{n+a}=-\varphi_{a}-\frac{2 \mathrm{i} \sin (\nu) \sinh \left(\theta_{a}\right) u_{a}}{\sinh \left(2 \lambda_{a}\right) \sinh \left(\mathrm{i} \nu-2 \lambda_{a}\right)}-\sum_{\substack{j=1 \\ j \neq a, n+a)}}^{N} \frac{\mathrm{i} \sin (\mu)\left(\sinh \left(\theta_{a}\right) u_{a}-\sinh \left(\Theta_{j}\right) u_{j}\right)}{\sinh \left(\lambda_{a}-\Lambda_{j}\right) \sinh \left(\mathrm{i} \mu-\lambda_{a}+\Lambda_{j}\right)}
$$

By invoking Proposition 1, let us observe that for the inverse of the matrix $L$ (3.6) we can write that $L^{-1}=C L C$, whence for the Hermitian matrix $L-L^{-1}$ we have

$$
\left(L-L^{-1}\right) C+C\left(L-L^{-1}\right)=L C-C L C^{2}+C L-C^{2} L C=0 .
$$

Thus, the matrix valued smooth function $\left(L-L^{-1}\right) / 2$ defined on the phase space $P(1.2)$ takes values in the subspace $\mathfrak{p}(2.7)$. Therefore, by taking its projection onto the Abelian subspace $\mathfrak{a}$ (2.10), we obtain the diagonal matrix

$$
D=\left(L-L^{-1}\right)_{\mathfrak{a}} / 2 \in \mathfrak{a}
$$

with diagonal entries

$$
D_{j, j}=\sinh \left(\Theta_{j}\right) u_{j} \quad\left(j \in \mathbb{N}_{N}\right) .
$$

Next, by projecting the function $\left(L-L^{-1}\right) / 2$ onto the complementary subspace $\mathfrak{a}^{\perp}$, we obtain the off-diagonal matrix

$$
Y=\left(L-L^{-1}\right)_{\mathfrak{a}^{\perp}} / 2 \in \mathfrak{a}^{\perp},
$$

which in turn allows us to introduce the matrix valued smooth function

$$
Z=\sinh \left(\widetilde{\operatorname{ad}_{\Lambda}}\right)^{-1} Y \in \mathfrak{m}^{\perp},
$$


too. Since $\lambda \in Q$ (1.1), the corresponding diagonal matrix $\Lambda$ (3.2) is regular in the sense that it takes values in the open Weyl chamber $\mathfrak{c} \subseteq \mathfrak{a}_{\text {reg }}$ (2.20). Therefore, $Z$ is indeed a well-defined off-diagonal $N \times N$ matrix, and its non-trivial entries take the form

$$
Z_{k, l}=\frac{Y_{k, l}}{\sinh \left(\Lambda_{k}-\Lambda_{l}\right)}=\frac{L_{k, l}-\left(L^{-1}\right)_{k, l}}{2 \sinh \left(\Lambda_{k}-\Lambda_{l}\right)} \quad\left(k, l \in \mathbb{N}_{N}, k \neq l\right) .
$$

Utilizing $Z$, for each $a \in \mathbb{N}_{n}$ we also define the function

$$
\mathcal{M}_{a}=\frac{\mathrm{i}}{F_{a}} \operatorname{Im}\left((Z F)_{a}\right)=\frac{\mathrm{i}}{F_{a}} \operatorname{Im}\left(\sum_{j=1}^{N} Z_{a, j} F_{j}\right) \in C^{\infty}(P) .
$$

Recalling the subspace $\mathfrak{m}(2.13)$, it is clear that

$$
B_{\mathfrak{m}}=\operatorname{diag}\left(\mathcal{M}_{1}, \ldots, \mathcal{M}_{n}, \mathcal{M}_{1}, \ldots, \mathcal{M}_{n}\right) \in \mathfrak{m}
$$

is a well-defined function. Having the above objects at our disposal, the content of Proposition 6] can be recast into a more convenient matrix form as follows.

Lemma 7. With the aid of the smooth functions $Z$ (4.41) and $B_{\mathfrak{m}}$ (4.44), for the derivative of the column vector $F$ (3.5) along the Hamiltonian vector field $\boldsymbol{X}_{H}$ (4.2) we can write

$$
\boldsymbol{X}_{H}[F]=\left(Z-B_{\mathfrak{m}}\right) F .
$$

Proof. Upon introducing the column vector

$$
J=\boldsymbol{X}_{H}[F]+B_{\mathfrak{m}} F-Z F \in \mathbb{C}^{N},
$$

it is enough to prove that $J_{k}=0$ for all $k \in \mathbb{N}_{N}$, at each point $(\lambda, \theta)$ of the phase space $P(1.2)$. Starting with the upper $n$ components of $J$, notice that by Proposition 6 and the formulae (4.42)-(4.44) we can write that

$$
J_{a}=\frac{1}{2} e^{-\frac{\theta_{a}}{2}} u_{a}^{\frac{3}{2}} G_{a} \quad\left(a \in \mathbb{N}_{n}\right)
$$

where $G_{a}$ is an appropriate function depending only on $\lambda$. More precisely, it has the form

$$
\begin{aligned}
G_{a}=\operatorname{Re}( & \frac{2 \mathrm{i} \sin (\nu)}{\sinh \left(2 \lambda_{a}\right) \sinh \left(\mathrm{i} \nu+2 \lambda_{a}\right)}+\sum_{\substack{j=1 \\
j \neq a, n+a)}}^{N} \frac{\mathrm{i} \sin (\mu)\left(1+\bar{z}_{j} \bar{z}_{a}^{-1}\right)}{\sinh \left(\lambda_{a}-\Lambda_{j}\right) \sinh \left(\mathrm{i} \mu+\lambda_{a}-\Lambda_{j}\right)} \\
& \left.+\frac{\mathrm{i} \sin (\mu)\left(z_{a} \bar{z}_{a}^{-1}-1\right)+\mathrm{i} \sin (\mu-\nu)\left(\bar{z}_{a}^{-1}-z_{a}^{-1}\right)}{\sinh \left(2 \lambda_{a}\right) \sinh \left(\mathrm{i} \mu+2 \lambda_{a}\right)}\right),
\end{aligned}
$$

that can be made quite explicit by exploiting the definition of the constituent functions $z_{j}(3.3)$. Now, following the same strategy we applied in the proof of Proposition 1, let us introduce a complex valued function $g_{a}$ depending only on a single complex variable $w$, obtained simply by replacing $\lambda_{a}$ with $\lambda_{a}+w$ in the explicit expression of right-hand side of the above equation (4.48). In mod $\mathrm{i} \pi$ sense this meromorphic function has at most first order poles at the points

$$
w \equiv-\lambda_{a}, w \equiv \pm \mathrm{i} \mu / 2-\lambda_{a}, w \equiv \pm \mathrm{i} \nu / 2-\lambda_{a}, w \equiv \Lambda_{j}-\lambda_{a}, w \equiv \pm\left(\mathrm{i} \mu+\Lambda_{j}\right)-\lambda_{a}\left(j \in \mathbb{N}_{N}\right) .
$$


However, at each of these points the residue of $g_{a}$ turns out to be zero. Moreover, it is obvious that $g_{a}(w)$ vanishes as $\operatorname{Re}(w) \rightarrow \infty$, therefore Liouville's theorem implies that $g_{a}(w)=0$ for all $w \in \mathbb{C}$. In particular $G_{a}=g_{a}(0)=0$, and so by (4.47) we conclude that $J_{a}=0$.

Turning to the lower $n$ components of the column vector $J$ (4.46), let us note that our previous result $J_{a}=0$ allows us to write that

$$
J_{n+a}=-\sinh \left(\theta_{a}\right) e^{-\frac{\theta_{a}}{2}} u_{a}^{\frac{1}{2}} \bar{z}_{a} G_{n+a} \quad\left(a \in \mathbb{N}_{n}\right)
$$

where $G_{n+a}$ is again an appropriate smooth function depending only on $\lambda$, as can be seen from the formula

$$
\begin{aligned}
G_{n+a}= & \frac{2 \mathrm{i} \sin (\nu)}{\sinh \left(2 \lambda_{a}\right) \sinh \left(\mathrm{i} \nu-2 \lambda_{a}\right)}-\frac{\mathrm{i} \sin (\mu)+\mathrm{i} \sin (\mu-\nu) \bar{z}_{a}^{-1}}{\sinh \left(2 \lambda_{a}\right) \sinh \left(\mathrm{i} \mu-2 \lambda_{a}\right)}+\bar{z}_{a}^{-1} \frac{\mathrm{i} \sin (\mu) z_{a}+\mathrm{i} \sin (\mu-\nu)}{\sinh \left(2 \lambda_{a}\right) \sinh \left(\mathrm{i} \mu+2 \lambda_{a}\right)} \\
& +\sum_{\substack{j=1 \\
j \neq a, n+a)}}^{N} \frac{1}{\sinh \left(\lambda_{a}-\Lambda_{j}\right)}\left(\frac{\mathrm{i} \sin (\mu)}{\sinh \left(\mathrm{i} \mu-\lambda_{a}+\Lambda_{j}\right)}+\frac{\mathrm{i} \sin (\mu) \bar{z}_{a}^{-1} \bar{z}_{j}}{\sinh \left(\mathrm{i} \mu+\lambda_{a}-\Lambda_{j}\right)}\right) .
\end{aligned}
$$

Next, let us plug the definition of $z_{j}$ (3.3) into the above expression (4.51) and introduce the complex valued function $g_{n+a}$ of $w \in \mathbb{C}$ by replacing $\lambda_{a}$ with $\lambda_{a}+w$ in the resulting formula. Note that $g_{n+a}$ has at most first order poles at the points

$$
w \equiv-\lambda_{a}, w \equiv \pm \mathrm{i} \mu / 2-\lambda_{a}, w \equiv \Lambda_{j}-\lambda_{a}\left(j \in \mathbb{N}_{N}\right) \quad(\bmod \mathrm{i} \pi)
$$

but all these singularities are removable. Since $g_{n+a}(w) \rightarrow 0$ as $\operatorname{Re}(w) \rightarrow \infty$, the boundedness of the periodic function $g_{n+a}$ is also obvious. Thus, Liouville's theorem entails that $g_{n+a}=0$ on the whole complex plane, whence the relationship $G_{n+a}=g_{n+a}(0)=0$ also follows. Now, looking back to the equation (4.50), we end up with the desired equation $J_{n+a}=0$.

\subsection{Lax representation of the dynamics}

Based on our proposed Lax matrix (3.6), in this subsection we wish to construct a Lax representation for the dynamics of the van Diejen system (1.5). As it turns out, Lemmas 3 and 7 prove to be instrumental in our approach. As the first step, by applying the Hamiltonian vector field $\boldsymbol{X}_{H}$ (4.2) on the Ruijsenaars type commutation relation (3.20), let us observe that the Leibniz rule yields

$$
\begin{aligned}
& e^{\mathrm{i} \mu} e^{\mathrm{ad}_{\boldsymbol{\Lambda}}}\left(\boldsymbol{X}_{H}[L]-\left[L, e^{-\boldsymbol{\Lambda}} \boldsymbol{X}_{H}\left[e^{\boldsymbol{\Lambda}}\right]\right]\right)-e^{-\mathrm{i} \mu} e^{-\mathrm{ad}_{\boldsymbol{\Lambda}}}\left(\boldsymbol{X}_{H}[L]+\left[L, \boldsymbol{X}_{H}\left[e^{\boldsymbol{\Lambda}}\right] e^{-\boldsymbol{\Lambda}}\right]\right) \\
& \quad=2 \mathrm{i} \sin (\mu)\left(\boldsymbol{X}_{H}[F] F^{*}+F\left(\boldsymbol{X}_{H}[F]\right)^{*}\right) .
\end{aligned}
$$

By comparing the formula appearing in (4.3) with the matrix entries (4.39) of the diagonal matrix $D$, it is clear that

$$
\boldsymbol{X}_{H}[\boldsymbol{\Lambda}]=D
$$

which in turn implies that

$$
e^{-\boldsymbol{\Lambda}} \boldsymbol{X}_{H}\left[e^{\boldsymbol{\Lambda}}\right]=\boldsymbol{X}_{H}\left[e^{\boldsymbol{\Lambda}}\right] e^{-\boldsymbol{\Lambda}}=D
$$


Thus, the above equation (4.53) can be cast into the fairly explicit form

$$
\begin{aligned}
& e^{\mathrm{i} \mu} e^{\mathrm{ad}_{\boldsymbol{\Lambda}}}\left(\boldsymbol{X}_{H}[L]-[L, D]\right)-e^{-\mathrm{i} \mu} e^{-\mathrm{ad}_{\boldsymbol{\Lambda}}}\left(\boldsymbol{X}_{H}[L]+[L, D]\right) \\
& \quad=2 \mathrm{i} \sin (\mu)\left(\boldsymbol{X}_{H}[F] F^{*}+F\left(\boldsymbol{X}_{H}[F]\right)^{*}\right),
\end{aligned}
$$

which serves as the starting point in our analysis on the derivative $\boldsymbol{X}_{H}[L]$. Before formulating the main result of this subsection, over the phase space $P(1.2)$ we define the matrix valued function

$$
B_{\mathfrak{m}^{\perp}}=-\operatorname{coth}\left(\widetilde{\operatorname{ad}_{\Lambda}}\right) Y \in \mathfrak{m}^{\perp} .
$$

Recalling the definition (4.40), we see that $B_{\mathfrak{m} \perp}$ is actually an off-diagonal matrix. Furthermore, for its non-trivial entries we have the explicit expressions

$$
\left(B_{\mathfrak{m}^{\perp}}\right)_{k, l}=-\operatorname{coth}\left(\Lambda_{k}-\Lambda_{l}\right) \frac{L_{k, l}-\left(L^{-1}\right)_{k, l}}{2} \quad\left(k, l \in \mathbb{N}_{N}, k \neq l\right) .
$$

Finally, with the aid of the diagonal matrix $B_{\mathfrak{m}}$ (4.44), over the phase space $P(1.2)$ we also define the $\mathfrak{k}$-valued smooth function

$$
B=B_{\mathfrak{m}}+B_{\mathfrak{m}^{\perp}} \in \mathfrak{k} .
$$

Theorem 8. The derivative of the matrix valued function $L$ (3.6) along the Hamiltonian vector field $\boldsymbol{X}_{H}$ (4.2) takes the Lax form

$$
\boldsymbol{X}_{H}[L]=[L, B] .
$$

In other words, the matrices $L$ (3.6) and $B$ (4.59) provide a Lax pair for the dynamics generated by the Hamiltonian (1.5).

Proof. For simplicity, let us introduce the matrix valued smooth functions

$$
\Psi=\boldsymbol{X}_{H}[L]-[L, B] \quad \text { and } \quad R=\sinh \left(\mathrm{i} \mu \operatorname{Id}_{\mathfrak{g l}(N, \mathbb{C})}+\operatorname{ad}_{\boldsymbol{\Lambda}}\right) \Psi
$$

defined on the phase space $P(1.2)$. Our goal is to prove that $\Psi=0$. However, $\operatorname{since} \sin (\mu) \neq 0$, the linear operator

$$
\sinh \left(\mathrm{i} \mu \operatorname{Id}_{\mathfrak{g l}(N, \mathbb{C})}+\operatorname{ad}_{\boldsymbol{\Lambda}}\right) \in \operatorname{End}(\mathfrak{g l}(N, \mathbb{C}))
$$

is invertible at each point of $P$, whence it is enough to show that $R=0$. For this reason, notice that from the relationship (4.56) we can infer that

$$
\begin{aligned}
2 R= & e^{\mathrm{i} \mu} e^{\operatorname{ad}_{\boldsymbol{\Lambda}}} \Psi-e^{-\mathrm{i} \mu} e^{-\operatorname{ad}_{\boldsymbol{\Lambda}}} \Psi \\
= & 2 \mathrm{i} \sin (\mu)\left(\boldsymbol{X}_{H}[F] F^{*}+F\left(\boldsymbol{X}_{H}[F]\right)^{*}\right)-\left(e^{\mathrm{i} \mu} e^{\operatorname{ad} \boldsymbol{\Lambda}}\left[L, B_{\mathfrak{m} \perp}\right]-e^{-\mathrm{i} \mu} e^{-\operatorname{ad}_{\boldsymbol{\Lambda}}}\left[L, B_{\mathfrak{m} \perp}\right]\right) \\
& -\left(e^{\mathrm{i} \mu} e^{\operatorname{ad}_{\boldsymbol{\Lambda}}}\left[L, B_{\mathfrak{m}}\right]-e^{-\mathrm{i} \mu} e^{-\operatorname{ad}_{\boldsymbol{\Lambda}}}\left[L, B_{\mathfrak{m}}\right]\right)-\left(e^{\mathrm{i} \mu} e^{\operatorname{ad}_{\boldsymbol{\Lambda}}}[D, L]+e^{-\mathrm{i} \mu} e^{-\operatorname{ad}_{\boldsymbol{\Lambda}}}[D, L]\right) .
\end{aligned}
$$

Our strategy is to inspect the right-hand side of the above equation term-by-term.

As a preparatory step, from the definitions of $D$ (4.38) and $Y$ (4.40) we see that

$$
\left(L-L^{-1}\right) / 2=D+Y,
$$

thus the commutation relation

$$
[L, Y]=\left[L,-D+\left(L-L^{-1}\right) / 2\right]=[D, L]
$$


readily follows. Keeping in mind the relationship (4.65) and the standard hyperbolic functional equations

$$
\operatorname{coth}(w) \pm 1=\frac{e^{ \pm w}}{\sinh (w)} \quad(w \in \mathbb{C})
$$

from the definitions of $Z$ (4.41) and $B_{\mathfrak{m}^{\perp}}$ (4.57) we infer that

$$
\begin{aligned}
e^{\operatorname{ad} \Lambda}\left[L, B_{\mathfrak{m}} \perp\right] & =-e^{\operatorname{ad}_{\Lambda}}\left[L, \operatorname{coth}\left(\widetilde{\operatorname{ad}_{\Lambda}}\right) Y\right] \\
& =-e^{\operatorname{ad}_{\Lambda}}\left(\left[L,\left(\operatorname{coth}(\widetilde{\operatorname{ad}} \boldsymbol{\Lambda})-\operatorname{Id}_{\mathfrak{m}^{\perp} \oplus \mathfrak{a}^{\perp}}\right) Y\right]+[L, Y]\right) \\
& =-e^{\operatorname{ad}_{\Lambda}}\left(\left[L, e^{-\widetilde{\operatorname{ad}}_{\Lambda}} \sinh (\widetilde{\operatorname{ad}})^{-1} Y\right]+[D, L]\right) \\
& =-\left[e^{\operatorname{ad}_{\Lambda}} L, Z\right]-e^{\operatorname{ad}_{\Lambda}}[D, L] .
\end{aligned}
$$

Along the same lines, one finds immediately that

$$
e^{-\operatorname{ad}_{\Lambda}}\left[L, B_{\mathfrak{m}^{\perp}}\right]=-\left[e^{-\operatorname{ad}_{\Lambda}} L, Z\right]+e^{-\operatorname{ad}_{\Lambda}}[D, L] .
$$

At this point let us recall that $Z$ (4.41) takes values in the subspace $\mathfrak{m}^{\perp} \subseteq \mathfrak{k}$, thus it is antiHermitian and commutes with the matrix $C$ (2.2). Therefore, by utilizing equations (4.67) and (4.68), the application of the commutation relation (3.20) leads to the relationship

$$
\begin{aligned}
e^{\mathrm{i} \mu} & e^{\operatorname{ad} \boldsymbol{\Lambda}}\left[L, B_{\mathfrak{m}^{\perp}}\right]-e^{-\mathrm{i} \mu} e^{-\operatorname{ad}_{\Lambda}}\left[L, B_{\mathfrak{m}^{\perp}}\right] \\
& =-\left[e^{\mathrm{i} \mu} e^{\operatorname{ad}_{\Lambda}} L-e^{-\mathrm{i} \mu} e^{-\operatorname{ad}_{\Lambda}} L, Z\right]-\left(e^{\mathrm{i} \mu} e^{\operatorname{ad}_{\boldsymbol{\Lambda}}}[D, L]+e^{-\mathrm{i} \mu} e^{-\operatorname{ad}_{\Lambda}}[D, L]\right) \\
& =-\left[2 \mathrm{i} \sin (\mu) F F^{*}+2 \mathrm{i} \sin (\mu-\nu) C, Z\right]-\left(e^{\mathrm{i} \mu} e^{\operatorname{ad}_{\Lambda}}[D, L]+e^{-\mathrm{i} \mu} e^{-\mathrm{ad}_{\boldsymbol{\Lambda}}}[D, L]\right) \\
& =2 \mathrm{i} \sin (\mu)\left((Z F) F^{*}+F(Z F)^{*}\right)-\left(e^{\mathrm{i} \mu} e^{\operatorname{ad}_{\Lambda}}[D, L]+e^{-\mathrm{i} \mu} e^{-\operatorname{ad}_{\boldsymbol{\Lambda}}}[D, L]\right) .
\end{aligned}
$$

To proceed further, let us recall that $B_{\mathfrak{m}}(4.44)$ takes values in $\mathfrak{m} \subseteq \mathfrak{k}$, whence it is also antiHermitian and also commutes with the matrix $C$ (2.2). Thus, by applying commutation relation (3.20) again, we obtain at once that

$$
\begin{aligned}
e^{\mathrm{i} \mu} & e^{\operatorname{ad}_{\Lambda}}\left[L, B_{\mathfrak{m}}\right]-e^{-\mathrm{i} \mu} e^{-\operatorname{ad}_{\Lambda}}\left[L, B_{\mathfrak{m}}\right] \\
& =e^{\mathrm{i} \mu}\left[e^{\operatorname{ad}_{\Lambda}} L, e^{\operatorname{ad}_{\Lambda}} B_{\mathfrak{m}}\right]-e^{-\mathrm{i} \mu}\left[e^{-\operatorname{ad}_{\Lambda}} L, e^{-\operatorname{ad}_{\Lambda}} B_{\mathfrak{m}}\right]=\left[e^{\mathrm{i} \mu} e^{\operatorname{ad}_{\Lambda}} L-e^{-\mathrm{i} \mu} e^{-\operatorname{ad}_{\Lambda}} L, B_{\mathfrak{m}}\right] \\
& =\left[2 \mathrm{i} \sin (\mu) F F^{*}+2 \mathrm{i} \sin (\mu-\nu) C, B_{\mathfrak{m}}\right]=-2 \mathrm{i} \sin (\mu)\left(\left(B_{\mathfrak{m}} F\right) F^{*}+F\left(B_{\mathfrak{m}} F\right)^{*}\right) .
\end{aligned}
$$

Now, by plugging the expressions (4.69) and (4.70) into (4.63), we obtain that

$$
R=\mathrm{i} \sin (\mu)\left(\left(\boldsymbol{X}_{H}[F]-Z F+B_{\mathfrak{m}} F\right) F^{*}+F\left(\boldsymbol{X}_{H}[F]-Z F+B_{\mathfrak{m}} F\right)^{*}\right) .
$$

Giving a glance at Lemma 7 , we conclude that $R=0$, thus the Theorem follows.

At this point we wish to make a short comment on matrix $B=B(\lambda, \theta ; \mu, \nu)$ (4.59) appearing in the Lax representation (4.60) of the dynamics (1.5). It is an important fact that by taking its 'rational limit' we can recover the second member of the Lax pair of the rational $C_{n}$ van Diejen system with two parameters $\mu$ and $\nu$. More precisely, up to some irrelevant numerical factors, in the $\alpha \rightarrow 0^{+}$limit the matrix $\alpha B(\alpha \lambda, \theta ; \alpha \mu, \alpha \nu)$ tends to the second member $\hat{\mathcal{B}}(\lambda, \theta ; \mu, \nu, \kappa=0)$ of the rational Lax pair, that first appeared in equation (4.60) of the recent paper [15]. In other 
words, matrix $B(4.59)$ is an appropriate hyperbolic generalization of the 'rational' matrix $\hat{\mathcal{B}}$ with two coupling parameters. We can safely state that the results presented in [15] has played a decisive role in our present work. As a matter of fact, most probably we could not have guessed the form of the non-trivial building blocks (4.44) and (4.57) without the knowledge of rational analogue of $B$.

In order to harvest some consequences of the Lax representation (4.60), we continue with a simple corollary of Theorem 8 , that proves to be quite handy in the developments of the next subsection.

Proposition 9. For the derivatives of the matrix valued smooth functions $D$ (4.38) and $Y$ (4.40) along the Hamiltonian vector field $\boldsymbol{X}_{H}$ (4.2) we have

$$
\boldsymbol{X}_{H}[D]=\left[Y, B_{\mathfrak{m}^{\perp}}\right]_{\mathfrak{a}} \quad \text { and } \quad \boldsymbol{X}_{H}[Y]=\left[Y, B_{\mathfrak{m}^{\perp}}\right]_{\mathfrak{a}^{\perp}}+\left[D, B_{\mathfrak{m}^{\perp}}\right]+\left[Y, B_{\mathfrak{m}}\right] .
$$

Proof. As a consequence of Proposition 1, for the inverse of $L$ we can write that $L^{-1}=C L C$. Since the matrix valued function $B$ (4.59) takes values in $\mathfrak{k}(2.6)$, from Theorem 8 we infer that

$$
\boldsymbol{X}\left[L^{-1}\right]=C \boldsymbol{X}_{H}[L] C=C[L, B] C=[C L C, C B C]=\left[L^{-1}, B\right],
$$

thus the equation

$$
\boldsymbol{X}_{H}\left[\left(L-L^{-1}\right) / 2\right]=\left[\left(L-L^{-1}\right) / 2, B\right]
$$

is immediate. Due to the relationship (4.64), by simply projecting of the above equation onto the subspaces $\mathfrak{a}$ and $\mathfrak{a}^{\perp}$, respectively, the derivatives displayed in (4.72) follow at once.

\subsection{Geodesic interpretation}

The geometric study of the CMS type integrable systems goes back to the fundamental works of Olshanetsky and Perelomov (see e.g. [9, 19]). Since their landmark papers the so-called projection method has been vastly generalized to cover many variants of the CMS type particle systems. By now some result are available in the context of the RSvD models, too. For details, see e.g. [20, 21, 22, 23, 10, 11]. The primary goal of this subsection is to show that the Hamiltonian flow generated by the Hamiltonian (1.5) can be also obtained by an appropriate 'projection method' from the geodesic flow of the Lie group $U(n, n)$. In order to make this statement more precise, take the maximal integral curve

$$
\mathbb{R} \ni t \mapsto(\lambda(t), \theta(t))=\left(\lambda_{1}(t), \ldots, \lambda_{n}(t), \theta_{1}(t), \ldots, \theta_{n}(t)\right) \in P
$$

of the Hamiltonian vector field $\boldsymbol{X}_{H}$ (4.2) satisfying the initial condition

$$
\gamma(0)=\gamma_{0}
$$

where $\gamma_{0} \in P$ is an arbitrary point. By exploiting Proposition 9, we start our analysis with the following observation.

Proposition 10. Along the maximally defined trajectory (4.75), the time evolution of the diagonal matrix $\boldsymbol{\Lambda}=\boldsymbol{\Lambda}(t) \in \mathfrak{c}$ (3.2) obeys the second order differential equation

$$
\ddot{\Lambda}+\left[Y, \operatorname{coth}\left(\widetilde{\operatorname{ad}}_{\boldsymbol{\Lambda}}\right) Y\right]_{\mathfrak{a}}=0,
$$


whilst for the evolution of $Y=Y(t)$ (4.40) we have the first order equation

$$
\dot{Y}+\left[Y, \operatorname{coth}\left(\widetilde{\operatorname{ad}}_{\boldsymbol{\Lambda}}\right) Y\right]_{\mathfrak{a}^{\perp}}-\left[Y, B_{\mathfrak{m}}\right]+\left[\dot{\boldsymbol{\Lambda}}, \operatorname{coth}\left(\widetilde{\operatorname{ad}}_{\boldsymbol{\Lambda}}\right) Y\right]=0
$$

Proof. Due to equation (4.54), along the solution curve (4.75) we can write

$$
\dot{\Lambda}=D
$$

whereas from the relationships displayed in (4.72) we get

$$
\dot{D}=\left[Y, B_{\mathfrak{m}^{\perp}}\right]_{\mathfrak{a}} \quad \text { and } \quad \dot{Y}=\left[Y, B_{\mathfrak{m}^{\perp}}\right]_{\mathfrak{a}^{\perp}}+\left[D, B_{\mathfrak{m}^{\perp}}\right]+\left[Y, B_{\mathfrak{m}}\right]
$$

Recalling the definition (4.57), equations (4.77) and (4.78) clearly follow.

Next, by evaluating the matrices $Z$ (4.41) and $B_{\mathfrak{m}}$ (4.44) along the fixed trajectory (4.75), for all $t \in \mathbb{R}$ we define

$$
\mathcal{K}(t)=B_{\mathfrak{m}}(t)-Z(t) \in \mathfrak{k} .
$$

Since the dependence of $\mathcal{K}$ on $t$ is smooth, there is a unique maximal smooth solution

$$
\mathbb{R} \ni t \mapsto k(t) \in G L(N, \mathbb{C})
$$

of the first order differential equation

$$
\dot{k}(t)=k(t) \mathcal{K}(t) \quad(t \in \mathbb{R})
$$

satisfying the initial condition

$$
k(0)=\mathbf{1}_{N} .
$$

Since (4.83) is a linear differential equation for $k$, the existence of such a global fundamental solution is obvious. Moreover, since $\mathcal{K}$ (4.81) takes values in the Lie algebra $\mathfrak{k}(2.6)$, the trivial observations

$$
\frac{\mathrm{d}\left(k C k^{*}\right)}{\mathrm{d} t}=\dot{k} C k^{*}+k C \dot{k}^{*}=k\left(\mathcal{K} C+C \mathcal{K}^{*}\right) k^{*}=0 \quad \text { and } \quad k(0) C k(0)^{*}=C
$$

imply immediately that $k(4.82)$ actually takes values in the subgroup $K(2.4)$; that is,

$$
k(t) \in K \quad(t \in \mathbb{R}) .
$$

Utilizing $k$, we can formulate the most important technical result of this subsection.

Lemma 11. The smooth function

$$
\mathbb{R} \ni t \mapsto A(t)=k(t) e^{2 \Lambda(t)} k(t)^{-1} \in \exp \left(\mathfrak{p}_{\text {reg }}\right)
$$

satisfies the second order geodesic differential equation

$$
\frac{\mathrm{d}}{\mathrm{d} t}\left(\frac{\mathrm{d} A(t)}{\mathrm{d} t} A(t)^{-1}\right)=0 \quad(t \in \mathbb{R}) .
$$


Proof. First, let us observe that (4.87) is a well-defined map. Indeed, since along the trajectory (4.75) we have $\Lambda(t) \in \mathfrak{c}$, from (2.22) we see that $A$ does take values in $\exp \left(\mathfrak{p}_{\text {reg }}\right)$. Continuing with the proof proper, notice that for all $t \in \mathbb{R}$ we have $A^{-1}=k e^{-2 \Lambda} k^{-1}$ and

$$
\dot{A}=\dot{k} e^{2 \boldsymbol{\Lambda}} k^{-1}+k e^{2 \boldsymbol{\Lambda}} 2 \dot{\boldsymbol{\Lambda}} k^{-1}-k e^{2 \boldsymbol{\Lambda}} k^{-1} \dot{k} k^{-1}
$$

thus the formulae

$$
\dot{A} A^{-1}=k\left(2 \dot{\Lambda}-e^{2 \operatorname{ad} \Lambda} \mathcal{K}+\mathcal{K}\right) k^{-1} \quad \text { and } \quad A^{-1} \dot{A}=k\left(2 \dot{\Lambda}+e^{-2 \operatorname{ad} \Lambda} \mathcal{K}-\mathcal{K}\right) k^{-1}
$$

are immediate. Upon introducing the shorthand notations

$$
\begin{aligned}
& \mathcal{L}(t)=\dot{\boldsymbol{\Lambda}}(t)+\cosh \left(\widetilde{\operatorname{ad}}_{\boldsymbol{\Lambda}(t)}\right) Y(t) \in \mathfrak{p} \\
& \mathcal{N}(t)=\sinh \left(\widetilde{\operatorname{ad}}_{\boldsymbol{\Lambda}(t)}\right) Y(t) \in \mathfrak{k}
\end{aligned}
$$

from (4.90) we conclude that

$$
\begin{aligned}
\frac{\dot{A} A^{-1}+A^{-1} \dot{A}}{4} & =k\left(\dot{\Lambda}-\frac{1}{2} \sinh \left(2 \operatorname{ad}_{\boldsymbol{\Lambda}}\right) \mathcal{K}\right) k^{-1}=k\left(\dot{\Lambda}-\frac{1}{2} \sinh \left(2 \widetilde{\operatorname{ad}}_{\boldsymbol{\Lambda}}\right) \mathcal{K}_{\mathfrak{m}^{\perp}}\right) k^{-1} \\
& =k\left(\dot{\boldsymbol{\Lambda}}+\cosh \left(\widetilde{\operatorname{ad}}_{\boldsymbol{\Lambda}}\right) \sinh \left(\widetilde{\operatorname{ad}_{\boldsymbol{\Lambda}}}\right) Z\right) k^{-1}=k \mathcal{L} k^{-1}
\end{aligned}
$$

and the relationship

$$
\begin{aligned}
\frac{\dot{A} A^{-1}-A^{-1} \dot{A}}{4} & =k \frac{\mathcal{K}-\cosh \left(2 \operatorname{ad}_{\boldsymbol{\Lambda}}\right) \mathcal{K}}{2} k^{-1}=-k\left(\sinh \left(\operatorname{ad}_{\boldsymbol{\Lambda}}\right)^{2} \mathcal{K}\right) k^{-1} \\
& =k\left(\sinh \left(\widetilde{\operatorname{ad}}_{\boldsymbol{\Lambda}}\right)^{2} Z\right) k^{-1}=k \mathcal{N} k^{-1}
\end{aligned}
$$

also follows.

Now, by differentiating (4.93) with respect to time $t$, we get

$$
\frac{\mathrm{d}}{\mathrm{d} t} \frac{\dot{A} A^{-1}+A^{-1} \dot{A}}{4}=k(\dot{\mathcal{L}}-[\mathcal{L}, \mathcal{K}]) k^{-1} .
$$

Recalling the definition (4.91), Leibniz rule yields

$$
\dot{\mathcal{L}}=\ddot{\Lambda}+\left[\dot{\Lambda}, \sinh \left(\widetilde{\operatorname{ad}}_{\Lambda}\right) Y\right]+\cosh \left(\widetilde{\operatorname{ad}}_{\Lambda}\right) \dot{Y}
$$

and the commutator

$$
[\mathcal{L}, \mathcal{K}]=-\left[\dot{\boldsymbol{\Lambda}}, \sinh \left(\widetilde{\operatorname{ad}}_{\boldsymbol{\Lambda}}\right)^{-1} Y\right]+\left[\cosh \left(\widetilde{\operatorname{ad}}_{\boldsymbol{\Lambda}}\right) Y, B_{\mathfrak{m}}\right]-\left[\cosh \left(\widetilde{\operatorname{ad}}_{\boldsymbol{\Lambda}}\right) Y, \sinh \left(\widetilde{\operatorname{ad}}_{\boldsymbol{\Lambda}}\right)^{-1} Y\right]
$$

is also immediate. By inspecting the right-hand side of the above equation, for the second term one can easily derive that

$$
\begin{aligned}
{\left[\cosh \left(\widetilde{\operatorname{ad}}_{\boldsymbol{\Lambda}}\right) Y, B_{\mathfrak{m}}\right] } & =\frac{1}{2}\left[e^{\operatorname{ad}_{\Lambda}} Y, B_{\mathfrak{m}}\right]+\frac{1}{2}\left[e^{-\operatorname{ad}_{\Lambda}} Y, B_{\mathfrak{m}}\right]=\frac{1}{2} e^{\operatorname{ad}_{\Lambda}}\left[Y, B_{\mathfrak{m}}\right]+\frac{1}{2} e^{-\operatorname{ad}_{\boldsymbol{\Lambda}}}\left[Y, B_{\mathfrak{m}}\right] \\
& =\cosh \left(\operatorname{ad}_{\boldsymbol{\Lambda}}\right)\left[Y, B_{\mathfrak{m}}\right]=\cosh (\widetilde{\operatorname{ad}} \boldsymbol{\Lambda})\left[Y, B_{\mathfrak{m}}\right] .
\end{aligned}
$$


Furthermore, bearing in mind the identities appearing in (4.66), a slightly longer calculation also reveals that the third term in (4.97) can be cast into the form

$$
\begin{aligned}
& {\left[\cosh \left(\widetilde{\operatorname{ad}}_{\boldsymbol{\Lambda}}\right) Y, \sinh \left(\widetilde{\operatorname{ad}}_{\boldsymbol{\Lambda}}\right)^{-1} Y\right]=\frac{1}{2}\left[e^{\operatorname{ad} \boldsymbol{\Lambda}} Y, \sinh \left(\widetilde{\operatorname{ad}}_{\boldsymbol{\Lambda}}\right)^{-1} Y\right]+\frac{1}{2}\left[e^{-\operatorname{ad}_{\boldsymbol{\Lambda}}} Y, \sinh \left(\widetilde{\operatorname{ad}}_{\boldsymbol{\Lambda}}\right)^{-1} Y\right]} \\
& =\frac{1}{2} e^{\operatorname{ad}_{\boldsymbol{\Lambda}}}\left[Y, e^{-\widetilde{\operatorname{ad}}_{\boldsymbol{\Lambda}}} \sinh \left(\widetilde{\operatorname{ad}}_{\boldsymbol{\Lambda}}\right)^{-1} Y\right]+\frac{1}{2} e^{-\operatorname{ad}_{\boldsymbol{\Lambda}}}\left[Y, e^{\widetilde{\operatorname{ad}}_{\boldsymbol{\Lambda}}} \sinh \left(\widetilde{\operatorname{ad}}_{\boldsymbol{\Lambda}}\right)^{-1} Y\right] \\
& =\cosh \left(\operatorname{ad}_{\boldsymbol{\Lambda}}\right)\left[Y, \operatorname{coth}\left(\widetilde{\operatorname{ad}}_{\boldsymbol{\Lambda}}\right) Y\right]=\left[Y, \operatorname{coth}\left(\widetilde{\operatorname{ad}}_{\boldsymbol{\Lambda}}\right) Y\right]_{\mathfrak{a}}+\cosh \left(\widetilde{\operatorname{ad}}_{\boldsymbol{\Lambda}}\right)\left[Y, \operatorname{coth}\left(\widetilde{\operatorname{ad}}_{\boldsymbol{\Lambda}}\right) Y\right]_{\mathfrak{a}^{\perp}} .
\end{aligned}
$$

Now, by plugging the expressions (4.98) and (4.99) into (4.97), and by applying the hyperbolic identity

$$
\sinh (w)+\frac{1}{\sinh (w)}=\cosh (w) \operatorname{coth}(w) \quad(w \in \mathbb{C}),
$$

one finds immediately that

$$
\begin{aligned}
\dot{\mathcal{L}}-[\mathcal{L}, \mathcal{K}]= & \ddot{\boldsymbol{\Lambda}}+\left[Y, \operatorname{coth}\left(\widetilde{\operatorname{ad}}_{\boldsymbol{\Lambda}}\right) Y\right]_{\mathfrak{a}} \\
& +\cosh \left(\widetilde{\operatorname{ad}}_{\boldsymbol{\Lambda}}\right)\left(\dot{Y}+\left[Y, \operatorname{coth}\left(\widetilde{\operatorname{ad}}_{\boldsymbol{\Lambda}}\right) Y\right]_{\mathfrak{a}^{\perp}}-\left[Y, B_{\mathfrak{m}}\right]+\left[\dot{\boldsymbol{\Lambda}}, \operatorname{coth}\left(\widetilde{\operatorname{ad}}_{\boldsymbol{\Lambda}}\right) Y\right]\right) .
\end{aligned}
$$

Looking back to Proposition [10, we see that $\dot{\mathcal{L}}-[\mathcal{L}, \mathcal{K}]=0$, thus by (4.95) we end up with the equation

$$
\frac{\mathrm{d}}{\mathrm{d} t} \frac{\dot{A} A^{-1}+A^{-1} \dot{A}}{4}=0 .
$$

Next, upon differentiating (4.94) with respect to $t$, we see that

$$
\frac{\mathrm{d}}{\mathrm{d} t} \frac{\dot{A} A^{-1}-A^{-1} \dot{A}}{4}=k(\dot{\mathcal{N}}-[\mathcal{N}, \mathcal{K}]) k^{-1} .
$$

Remembering the form of $\mathcal{N}$ (4.92), Leibniz rule yields

$$
\dot{\mathcal{N}}=\cosh \left(\widetilde{\operatorname{ad}}_{\boldsymbol{\Lambda}}\right)[\dot{\boldsymbol{\Lambda}}, Y]+\sinh \left(\widetilde{\operatorname{ad}}_{\boldsymbol{\Lambda}}\right) \dot{Y}=\sinh \left(\widetilde{\operatorname{ad}}_{\boldsymbol{\Lambda}}\right)\left(\operatorname{coth}\left(\widetilde{\operatorname{ad}}_{\boldsymbol{\Lambda}}\right)[\dot{\boldsymbol{\Lambda}}, Y]+\dot{Y}\right)
$$

and the formula

$$
[\mathcal{N}, \mathcal{K}]=\left[\sinh \left(\widetilde{\operatorname{ad}}_{\boldsymbol{\Lambda}}\right) Y, B_{\mathfrak{m}}\right]-\left[\sinh \left(\widetilde{\operatorname{ad}}_{\boldsymbol{\Lambda}}\right) Y, \sinh \left(\widetilde{\operatorname{ad}}_{\boldsymbol{\Lambda}}\right)^{-1} Y\right]
$$

is also immediate. Now, let us observe that the first term on the right-hand side of the above equation can be transformed into the equivalent form

$$
\begin{aligned}
{\left[\sinh \left(\widetilde{\operatorname{ad}}_{\boldsymbol{\Lambda}}\right) Y, B_{\mathfrak{m}}\right] } & =\frac{1}{2}\left[e^{\operatorname{ad}_{\Lambda}} Y, B_{\mathfrak{m}}\right]-\frac{1}{2}\left[e^{-\operatorname{ad}_{\Lambda}} Y, B_{\mathfrak{m}}\right]=\frac{1}{2} e^{\operatorname{ad} \boldsymbol{\Lambda}}\left[Y, B_{\mathfrak{m}}\right]-\frac{1}{2} e^{-\operatorname{ad}_{\Lambda}}\left[Y, B_{\mathfrak{m}}\right] \\
& =\sinh \left(\operatorname{ad}_{\boldsymbol{\Lambda}}\right)\left[Y, B_{\mathfrak{m}}\right]=\sinh \left(\widetilde{\operatorname{ad}}_{\boldsymbol{\Lambda}}\right)\left[Y, B_{\mathfrak{m}}\right]
\end{aligned}
$$

while for the second term we get

$$
\begin{aligned}
& {\left[\sinh \left(\widetilde{\operatorname{ad}}_{\boldsymbol{\Lambda}}\right) Y, \sinh \left(\widetilde{\operatorname{ad}}_{\boldsymbol{\Lambda}}\right)^{-1} Y\right]=\frac{1}{2}\left[e^{\operatorname{ad} \Lambda} Y, \sinh \left(\widetilde{\operatorname{ad}}_{\boldsymbol{\Lambda}}\right)^{-1} Y\right]-\frac{1}{2}\left[e^{-\operatorname{ad}_{\Lambda}} Y, \sinh \left(\widetilde{\operatorname{ad}}_{\boldsymbol{\Lambda}}\right)^{-1} Y\right]} \\
& \quad=\frac{1}{2} e^{\operatorname{ad}_{\boldsymbol{\Lambda}}}\left[Y, e^{-\widetilde{\operatorname{ad}}_{\boldsymbol{\Lambda}}} \sinh \left(\widetilde{\operatorname{ad}}_{\boldsymbol{\Lambda}}\right)^{-1} Y\right]-\frac{1}{2} e^{-\widetilde{a d}_{\boldsymbol{\Lambda}}}\left[Y, e^{\widetilde{\operatorname{ad}}_{\boldsymbol{\Lambda}}} \sinh \left(\widetilde{\operatorname{ad}}_{\boldsymbol{\Lambda}}\right)^{-1} Y\right] \\
& \quad=\sinh \left(\operatorname{ad}_{\boldsymbol{\Lambda}}\right)\left[Y, \operatorname{coth}\left(\widetilde{\operatorname{ad}}_{\boldsymbol{\Lambda}}\right) Y\right]=\sinh (\widetilde{\operatorname{ad}} \boldsymbol{\Lambda})\left[Y, \operatorname{coth}\left(\widetilde{\operatorname{ad}}_{\boldsymbol{\Lambda}}\right) Y\right]_{\mathfrak{a}^{\perp}} .
\end{aligned}
$$


Taking into account the above expressions, we obtain that

$$
\dot{\mathcal{N}}-[\mathcal{N}, \mathcal{K}]=\sinh \left(\widetilde{\operatorname{ad}}_{\boldsymbol{\Lambda}}\right)\left(\dot{Y}+\left[Y, \operatorname{coth}\left(\widetilde{\operatorname{ad}}_{\boldsymbol{\Lambda}}\right) Y\right]_{\mathfrak{a}^{\perp}}-\left[Y, B_{\mathfrak{m}}\right]+\left[\dot{\boldsymbol{\Lambda}}, \operatorname{coth}\left(\widetilde{\operatorname{ad}}_{\boldsymbol{\Lambda}}\right) Y\right]\right),
$$

whence by Proposition [10 we are entitled to write that $\dot{\mathcal{N}}-[\mathcal{N}, \mathcal{K}]=0$. Giving a glance at the relationship (4.103), it readily follows that

$$
\frac{\mathrm{d}}{\mathrm{d} t} \frac{\dot{A} A^{-1}-A^{-1} \dot{A}}{4}=0 .
$$

To complete the proof, observe that the desired geodesic equation (4.88) is a trivial consequence of the equations (4.102) and (4.109).

To proceed further, let us observe that by integrating the differential equation (4.88), we obtain immediately that

$$
\dot{A}(t) A(t)^{-1}=\dot{A}(0) A(0)^{-1} \quad(t \in \mathbb{R}) .
$$

However, recalling the definitions (4.91) and (4.92), and also the relationships (4.79) and (4.64), from the equations (4.93), (4.94) and (4.84) we infer that

$$
\begin{aligned}
\dot{A}(0) A(0)^{-1} & =2 k(0)(\mathcal{L}(0)+\mathcal{N}(0)) k(0)^{-1}=2\left(\dot{\boldsymbol{\Lambda}}(0)+e^{\operatorname{ad}_{\boldsymbol{\Lambda}(0)} Y(0)}\right) \\
& =2 e^{\operatorname{ad}_{\boldsymbol{\Lambda}(0)}}(D(0)+Y(0))=e^{\boldsymbol{\Lambda}(0)}\left(L(0)-L(0)^{-1}\right) e^{-\boldsymbol{\Lambda}(0)}
\end{aligned}
$$

Moreover, remembering (4.84) and the definition (4.87), at $t=0$ we can also write that

$$
A(0)=k(0) e^{2 \boldsymbol{\Lambda}(0)} k(0)^{-1}=e^{2 \boldsymbol{\Lambda}(0)} .
$$

Putting the above observations together, it is now evident that the unique maximal solution of the first order differential equation (4.110) with the initial condition (4.112) is the smooth curve

$$
A(t)=e^{t e^{\boldsymbol{\Lambda}(0)}\left(L(0)-L(0)^{-1}\right) e^{-\boldsymbol{\Lambda}(0)}} e^{2 \boldsymbol{\Lambda}(0)}=e^{\boldsymbol{\Lambda}(0)} e^{t\left(L(0)-L(0)^{-1}\right)} e^{\boldsymbol{\Lambda}(0)} \quad(t \in \mathbb{R}) .
$$

Comparing this formula with (4.87), the following result is immediate.

Theorem 12. Take an arbitrary maximal solution (4.75) of the van Diejen system (1.5), then at each $t \in \mathbb{R}$ it can be recovered uniquely from the spectral identification

$$
\left\{e^{ \pm 2 \lambda_{a}(t)} \mid a \in \mathbb{N}_{n}\right\}=\operatorname{Spec}\left(e^{\boldsymbol{\Lambda}(0)} e^{t\left(L(0)-L(0)^{-1}\right)} e^{\mathbf{\Lambda}(0)}\right) .
$$

The essence of the above theorem is that any solution (4.75) of the van Diejen system (1.5) can be obtained by a purely algebraic process based on the diagonalization of a matrix flow. Indeed, once one finds the evolution of $\lambda(t)$ from (4.114), the evolution of $\theta(t)$ also becomes accessible by the formula

$$
\theta_{a}(t)=\operatorname{arcsinh}\left(\frac{\dot{\lambda}_{a}(t)}{u_{a}(\lambda(t))}\right) \quad\left(a \in \mathbb{N}_{n}\right),
$$

as dictated by the equation of motion (4.3). 


\subsection{Temporal asymptotics}

One of the immediate consequences of the projection method formulated in the previous subsection is that the Hamiltonian (1.5) describes a 'repelling' particle system, thus it is fully justified to inquire about its scattering properties. Although rigorous scattering theory is in general a hard subject, a careful study of the algebraic solution algorithm described in Theorem 12 allows us to investigate the asymptotic properties of any maximally defined trajectory (4.75) as $t \rightarrow \pm \infty$. In this respect our main tool is Ruijsenaars' theorem on the spectral asymptotics of exponential type matrix flows (see Theorem A2 in [17]). To make it work, let us look at the relationship (3.24) and Lemma 4, from where we see that there is a group element $y \in K$ and a unique real $n$-tuple $\hat{\theta}=\left(\hat{\theta}_{1}, \ldots, \hat{\theta}_{n}\right) \in \mathbb{R}^{n}$ satisfying

$$
\hat{\theta}_{1}>\ldots>\hat{\theta}_{n}>0
$$

such that with the (regular) diagonal matrix $\hat{\boldsymbol{\Theta}} \in \mathfrak{c}$ defined in (3.23) we can write that

$$
L(0)=y e^{2 \hat{\mathbf{\Theta}}} y^{-1}
$$

Following the notations of the previous subsection, here $L(0)$ still stands for the Lax matrix (3.6) evaluated along the trajectory (4.75) at $t=0$. Since

$$
L(0)-L(0)^{-1}=2 y \sinh (2 \hat{\mathbf{\Theta}}) y^{-1}
$$

with the aid of the positive definite matrix

$$
\hat{L}=y^{-1} e^{2 \Lambda(0)} y \in \exp (\mathfrak{p})
$$

for the spectrum of the matrix flow appearing in (4.113) we obtain at once that

$$
\operatorname{Spec}\left(e^{\mathbf{\Lambda}(0)} e^{t\left(L(0)-L(0)^{-1}\right)} e^{\mathbf{\Lambda}(0)}\right)=\operatorname{Spec}\left(\hat{L} e^{2 t \sinh (2 \hat{\mathbf{\Theta}})}\right) .
$$

In order to make a closer contact with Ruijsenaars' theorem, let us also introduce the Hermitian $n \times n$ matrix $\mathcal{R}$ with entries

$$
\mathcal{R}_{a, b}=\delta_{a+b, n+1} .
$$

Since $\mathcal{R}^{2}=\mathbf{1}_{n}$, we have $\mathcal{R}^{-1}=\mathcal{R}$, whence the block-diagonal matrix

$$
\mathcal{W}=\left[\begin{array}{cc}
\mathbf{1}_{n} & 0_{n} \\
0_{n} & \mathcal{R}_{n}
\end{array}\right] \in G L(N, \mathbb{C}),
$$

also satisfies the relations $\mathcal{W}^{-1}=\mathcal{W}=\mathcal{W}^{*}$. As the most important ingredients of our present analysis, now we introduce the matrices

$$
\Theta^{+}=2 \mathcal{W} \hat{\Theta} \mathcal{W}^{-1} \text { and } \quad \tilde{L}=\mathcal{W} \hat{L} \mathcal{W}^{-1} \text {. }
$$

Recalling the relationships (4.114) and (4.120), it is clear that for all $t \in \mathbb{R}$ we can write that

$$
\left\{e^{ \pm 2 \lambda_{a}(t)} \mid a \in \mathbb{N}_{n}\right\}=\operatorname{Spec}\left(\tilde{L} e^{2 t \sinh \left(\Theta^{+}\right)}\right) .
$$


However, upon performing the conjugations with the unitary matrix $\mathcal{W}$ (4.122) in the defining equations displayed in (4.123), we find immediately that

$$
\Theta^{+}=\operatorname{diag}\left(\theta_{1}^{+}, \ldots, \theta_{n}^{+},-\theta_{n}^{+}, \ldots,-\theta_{1}^{+}\right)
$$

where

$$
\theta_{a}^{+}=2 \hat{\theta}_{a} \quad\left(a \in \mathbb{N}_{n}\right) .
$$

The point is that, due to our regularity result formulated in Lemma 4, the diagonal matrix (4.125) has a simple spectrum, and its eigenvalues are in strictly decreasing order along the diagonal (see (4.116)). Moreover, since $\hat{L}(4.119)$ is positive definite, so is $\tilde{L}$. In particular, the leading principal minors of matrix $\tilde{L}$ are all strictly positive. So, the exponential type matrix flow

$$
\mathbb{R} \ni t \mapsto \tilde{L} e^{2 t \sinh \left(\Theta^{+}\right)} \in G L(N, \mathbb{C})
$$

does meet all the requirements of Ruijsenaars' aforementioned theorem. Therefore, essentially by taking the logarithm of the quotients of the consecutive leading principal minors of the $n \times n$ submatrix taken from the upper-left-hand corner of $\tilde{L}$, one finds a unique real $n$-tuple

$$
\lambda^{+}=\left(\lambda_{1}^{+}, \ldots, \lambda_{n}^{+}\right) \in \mathbb{R}^{n}
$$

such that for all $a \in \mathbb{N}_{n}$ we can write

$$
\lambda_{a}(t) \sim t \sinh \left(\theta_{a}^{+}\right)+\lambda_{a}^{+} \text {and } \theta_{a}(t) \sim \theta_{a}^{+},
$$

up to exponentially vanishing small terms as $t \rightarrow \infty$. It is obvious that the same ideas work for the case $t \rightarrow-\infty$, too, with complete control over the asymptotic momenta $\theta_{a}^{-}$and the asymptotic phases $\lambda_{a}^{-}$as well. The above observations can be summarized as follows.

Lemma 13. For an arbitrary maximal solution (4.75) of the hyperbolic n-particle van Diejen system (1.5) the particles move asymptotically freely as $|t| \rightarrow \infty$. More precisely, for all $a \in \mathbb{N}_{n}$ we have the asymptotics

$$
\lambda_{a}(t) \sim t \sinh \left(\theta_{a}^{ \pm}\right)+\lambda_{a}^{ \pm} \quad \text { and } \quad \theta_{a}(t) \sim \theta_{a}^{ \pm} \quad(t \rightarrow \pm \infty),
$$

where the asymptotic momenta obey

$$
\theta_{a}^{-}=-\theta_{a}^{+} \quad \text { and } \quad \theta_{1}^{+}>\ldots>\theta_{n}^{+}>0 .
$$

We find it quite remarkable that, up to an overall sign, the asymptotic momenta are preserved (4.131). Following Ruijsenaars' terminology [17, 24], we may say that the 2-parameter family of van Diejen systems (1.5) are finite dimensional pure soliton systems. Now, let us remember that for each pure soliton system analyzed in the earlier literature, the scattering map has a factorized form. That is, the $n$-particle scattering can be completely reconstructed from the 2-particle processes, and also by the 1-particle scattering on the external potential (see e.g. [25, 26, 17, 24, 12]). Albeit the results we shall present in rest of the paper do not rely on this peculiar feature of the scattering process, still, it would be of considerable interest to prove this property for the hyperbolic van Diejen systems (1.5), too. However, because of its subtleties, we wish to work out the details of the scattering theory in a later publication. 


\section{Spectral invariants of the Lax matrix}

The ultimate goal of this section is to prove that the eigenvalues of the Lax matrix $L(\underline{3.6})$ are in involution. Superficially, one could say that it follows easily from the scattering theoretical results presented in the previous section. A convincing argument would go as follows. Recalling the notations (4.75) and (4.76), let us consider the flow

$$
\Phi: \mathbb{R} \times P \rightarrow P, \quad\left(t, \gamma_{0}\right) \mapsto \Phi_{t}\left(\gamma_{0}\right)=\gamma(t)
$$

generated by the Hamiltonian vector field $\boldsymbol{X}_{H}$ (4.2). Since for all $t \in \mathbb{R}$ the map $\Phi_{t}: P \rightarrow P$ is a symplectomorphism, for all $a, b \in \mathbb{N}_{n}$ we can write that

$$
\left\{\theta_{a} \circ \Phi_{t}, \theta_{b} \circ \Phi_{t}\right\}=\left\{\theta_{a}, \theta_{b}\right\} \circ \Phi_{t}=0 .
$$

On the other hand, from (4.130) it is also clear that at each point of the phase space $P$, for all $c \in \mathbb{N}_{n}$ we have

$$
\theta_{c} \circ \Phi_{t} \rightarrow \theta_{c}^{+} \quad(t \rightarrow \infty) .
$$

Recalling (3.44) and (4.126), it is evident that $\theta_{c}^{+} \in C^{\infty}(P)$. Therefore, by a 'simple interchange of limits', from (5.2) and (5.3) one could infer that the asymptotic momenta $\theta_{c}^{+}\left(c \in \mathbb{N}_{n}\right)$ Poisson commute. Bearing in mind the relationships (4.126) and (3.36), it would also follow that the eigenvalues of $L(3.6)$ generate a maximal Abelian Poisson subalgebra. However, to justify the interchange of limits, one does need a deeper knowledge about the scattering properties than the pointwise limit formulated in (5.3). Since we wish to work out the full scattering theory elsewhere, in this paper we choose an alternative approach by merging the temporal asymptotics of the trajectories with van Diejen's earlier results [1, 2, 3].

\subsection{Link to the 5-parameter family of van Diejen systems}

As is known from the seminal papers [2, 3], the definition of the classical hyperbolic van Diejen system is based on the smooth functions $v, w: \mathbb{R} \backslash\{0\} \rightarrow \mathbb{C}$ defined by the formulae

$$
v(x)=\frac{\sinh (\mathrm{i} g+x)}{\sinh (x)}, \quad w(x)=\frac{\sinh \left(\mathrm{i} g_{0}+x\right)}{\sinh (x)} \frac{\cosh \left(\mathrm{i} g_{1}+x\right)}{\cosh (x)} \frac{\sinh \left(\mathrm{i} g_{0}^{\prime}+x\right)}{\sinh (x)} \frac{\cosh \left(\mathrm{i} g_{1}^{\prime}+x\right)}{\cosh (x)},
$$

where the five independent real numbers $g, g_{0}, g_{1}, g_{0}^{\prime}, g_{1}^{\prime}$ are the coupling constants. Parameter $g$ in the 'potential' function $v$ controls the strength of inter-particle interaction, whereas the remaining four constants appearing in the 'external potential' $w$ are responsible for the influence of the ambient field. Conforming to the notations introduced in the aforementioned papers, let us recall that the set of Poisson commuting functions found by van Diejen can be succinctly written as

$$
H_{l}=\sum_{\substack{J \subseteq \mathbb{N}_{n},|J| \leq l \\ \varepsilon_{j}= \pm 1, j \in J}} \cosh \left(\theta_{\varepsilon J}\right)\left|V_{\varepsilon J ; J^{c}}\right| U_{J^{c}, l-|J|} \quad\left(l \in \mathbb{N}_{n}\right),
$$

where the various constituents are defined by the formulae

$$
\theta_{\varepsilon J}=\sum_{j \in J} \varepsilon_{j} \theta_{j}, \quad V_{\varepsilon J ; J^{c}}=\prod_{j \in J} w\left(\varepsilon_{j} \lambda_{j}\right) \prod_{\substack{j, j^{\prime} \in J \\\left(j<j^{\prime}\right)}} v\left(\varepsilon_{j} \lambda_{j}+\varepsilon_{j^{\prime}} \lambda_{j^{\prime}}\right)^{2} \prod_{\substack{j \in J \\ k \in J^{c}}} v\left(\varepsilon_{j} \lambda_{j}+\lambda_{k}\right) v\left(\varepsilon_{j} \lambda_{j}-\lambda_{k}\right),
$$


together with the expression

$$
U_{J^{c}, l-|J|}=(-1)^{l-|J|} \sum_{\substack{I \subseteq J^{c},|I|=l-|J| \\ \varepsilon_{i}= \pm 1, i \in I}} \prod_{i \in I} w\left(\varepsilon_{i} \lambda_{i}\right) \prod_{\substack{i, i^{\prime} \in I \\\left(i<i^{\prime}\right)}}\left|v\left(\varepsilon_{i} \lambda_{i}+\varepsilon_{i^{\prime}} \lambda_{i^{\prime}}\right)\right|^{2} \prod_{\substack{i \in I \\ k \in J^{c} \backslash I}} v\left(\varepsilon_{i} \lambda_{i}+\lambda_{k}\right) v\left(\varepsilon_{i} \lambda_{i}-\lambda_{k}\right) .
$$

At this point two short technical remarks are in order. First, we extend the family of the first integrals (5.5) with the constant function $H_{0}=1$. Analogously, in the last equation (5.7) it is understood that $U_{J^{c}, 0}=1$.

To make contact with the 2-parameter family of van Diejen systems of our interest (1.5), for the coupling parameters of the potential functions (5.4) we make the special choice

$$
g=\mu, \quad g_{0}=g_{1}=\frac{\nu}{2}, \quad g_{0}^{\prime}=g_{1}^{\prime}=0 .
$$

Under this assumption, from the definitions (3.3) and (5.6) it is evident that with the singleton $J=\{a\}$ we can write that

$$
V_{\{a\} ;\{a\}^{c}}=-z_{a} \quad\left(a \in \mathbb{N}_{n}\right) .
$$

Giving a glance at (5.7), it is also clear that the term corresponding to $J=\emptyset$ in the defining sum of $H_{1}$ (5.5) is a constant function of the form

$$
U_{\mathbb{N}_{n}, 1}=2 \sum_{a=1}^{n} \operatorname{Re}\left(z_{a}\right)=-2 \cos (\nu+(n-1) \mu) \frac{\sin (n \mu)}{\sin (\mu)} .
$$

Plugging the above formulae into van Diejen's main Hamiltonian $H_{1}$ (5.5), one finds immediately that

$$
H_{1}+2 \cos (\nu+(n-1) \mu) \frac{\sin (n \mu)}{\sin (\mu)}=2 H=\operatorname{tr}(L) .
$$

That is, up to some irrelevant constants, our Hamiltonian $H$ (1.5) can be identified with $H_{1}$ (5.5), provided the coupling parameters are related by the equations displayed in (5.8). At this point one may suspect that the quantities $\operatorname{tr}\left(L^{l}\right)$ are also expressible with the aid of the Poisson commuting family of functions $H_{l}$ (5.5). Clearly, it would imply immediately that the eigenvalues of the Lax matrix $L(3.6)$ are in involution. However, due to the complexity of the underlying objects (5.6)-(5.7), this naive approach would lead to a formidable combinatorial task, that we do not wish to pursue in this paper. To circumvent the difficulties, below we rather resort to a clean analytical approach by exploiting the scattering theoretical results formulated in the previous section.

\subsection{Poisson brackets of the eigenvalues of $L$}

Take an arbitrary point $\gamma_{0} \in P$ and consider the unique maximal integral curve

$$
\mathbb{R} \ni t \mapsto \gamma(t)=(\lambda(t), \theta(t)) \in P
$$

of the Hamiltonian vector field $\boldsymbol{X}_{H}$ (4.2) satisfying the initial condition

$$
\gamma(0)=\gamma_{0}
$$


Since the functions $H_{l}$ (5.5) are first integrals of the dynamics, their values at the point $\gamma_{0}$ can be recovered by inspecting the limit of $H_{l}(\gamma(t))$ as $t \rightarrow \infty$. Now, recalling the potentials (5.5) and the specialization of the coupling parameters (5.8), it is evident that

$$
\lim _{x \rightarrow \pm \infty} v(x)=e^{ \pm \mathrm{i} \mu} \quad \text { and } \quad \lim _{x \rightarrow \pm \infty} w(x)=e^{ \pm \mathrm{i} \nu} .
$$

Therefore, taking into account the regularity properties (4.131) of the asymptotic momenta $\theta_{c}^{+}$ (4.130), from Lemma 13 and the definitions (5.5)-(5.7) one finds immediately that

$$
H_{l}\left(\gamma_{0}\right)=\lim _{t \rightarrow \infty} H_{l}(\gamma(t))=\sum_{\substack{J \subseteq \mathbb{N}_{n},|J| \leq l \\ \varepsilon_{j}= \pm 1, j \in J}} \cosh \left(\theta_{\varepsilon J}^{+}\right) \mathcal{U}_{J^{c}, l-|J|} \quad\left(l \in \mathbb{N}_{n}\right),
$$

where

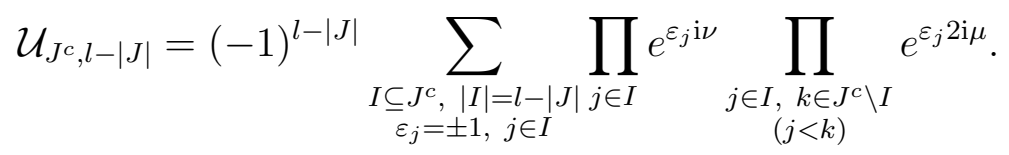

By inspecting the above expression, let us observe that the value of $\mathcal{U}_{J^{c}, l-|J|}$ does not depend on the specific choice of the subset $J$, but only on its cardinality $|J|$. More precisely, if $J \subseteq \mathbb{N}_{n}$ is an arbitrary subset of cardinality $|J|=k(0 \leq k \leq l-1)$, then we can write that

$$
\mathcal{U}_{J^{c}, l-|J|}=(-1)^{l-k} \sum_{\substack{1 \leq j_{1}<\cdots<j_{l-k} \leq n-k \\ \varepsilon_{1}= \pm 1, \ldots, \varepsilon_{l-k}= \pm 1}} \exp \left(\mathrm{i} \sum_{m=1}^{l-k} \varepsilon_{m}\left(\nu+2\left(n-l+m-j_{m}\right) \mu\right)\right) .
$$

To proceed further, let us now turn to the study of the Lax matrix $L$ (3.6). Due to the Lax representation of the dynamics that we established in Theorem 8 , the eigenvalues of $L$ are conserved quantities. Consequently, the coefficients $K_{0}, K_{1}, \ldots, K_{N} \in C^{\infty}(P)$ of the characteristic polynomial

$$
\operatorname{det}\left(L-y \mathbf{1}_{N}\right)=\sum_{m=0}^{N} K_{N-m} y^{m} \quad(y \in \mathbb{C})
$$

are also first integrals. As expected, the special algebraic properties of $L$ formulated in Proposition 1 and Lemma 2 have a profound impact on these coefficients as well, as can be seen from the relations

$$
K_{N-m}=K_{m} \quad(m=0,1, \ldots, N) .
$$

So, it is enough to analyze the properties of the members $K_{0}=1, K_{1}, \ldots, K_{n}$. In this respect the most important ingredient is the relationship

$$
\lim _{t \rightarrow \infty} L(\gamma(t))=\exp \left(\boldsymbol{\Theta}^{+}\right),
$$

where $\Theta^{+}$is the $N \times N$ diagonal matrix (4.125) containing the asymptotic momenta. Therefore, looking back to the definition (5.18), for any $m=0,1, \ldots, n$ we obtain at once that

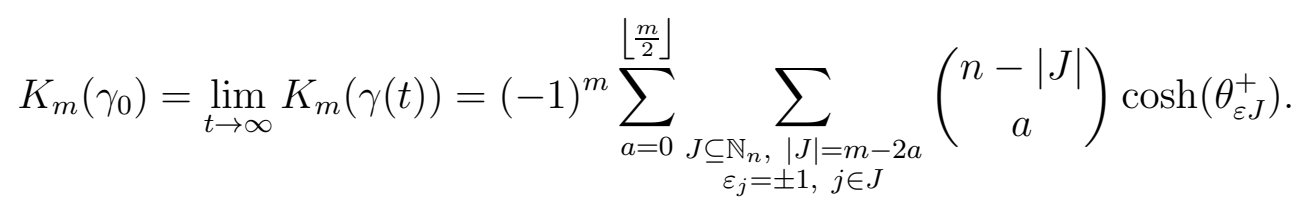

Based on the formulae (5.15) and (5.21), we can prove the following important technical result. 
Lemma 14. The two distinguished families of first integrals $\left\{H_{l}\right\}_{l=0}^{n}$ and $\left\{K_{m}\right\}_{m=0}^{n}$ are connected by an invertible linear relation with purely numerical coefficients depending only on the coupling parameters $\mu$ and $\nu$.

Proof. For brevity, let us introduce the notation

$$
\mathcal{A}_{k}=\sum_{\substack{J \subseteq \mathbb{N}_{n},|J|=k \\ \varepsilon_{j}= \pm 1, j \in J}} \cosh \left(\theta_{\varepsilon J}^{+}\right) \quad(k=0,1, \ldots, n) .
$$

As we have seen in (5.17), the coefficients $\mathcal{U}_{J^{c}, l-|J|}$ appearing in the formula (5.15) depend only on the cardinality of $J$, whence for any $l \in\{0,1, \ldots, n\}$ we can write that

$$
H_{l}\left(\gamma_{0}\right)=\sum_{k=0}^{l} \mathcal{U}_{\mathbb{N}_{n-k}, l-k} \mathcal{A}_{k}
$$

Since $\mathcal{U}_{\mathbb{N}_{n-l}, 0}=1$, the matrix transforming $\left\{\mathcal{A}_{k}\right\}_{k=0}^{n}$ into $\left\{H_{l}\left(\gamma_{0}\right)\right\}_{l=0}^{n}$ is lower triangular with plus ones on the diagonal, whence the above linear relation (5.23) is invertible. Comparing the formulae (5.21) and (5.22), it is also clear that

$$
K_{m}\left(\gamma_{0}\right)=(-1)^{m} \sum_{a=0}^{\left\lfloor\frac{m}{2}\right\rfloor}\left(\begin{array}{c}
n-(m-2 a) \\
a
\end{array}\right) \mathcal{A}_{m-2 a}
$$

which in turn implies that the matrix relating $\left\{\mathcal{A}_{k}\right\}_{k=0}^{n}$ to $\left\{K_{m}\left(\gamma_{0}\right)\right\}_{m=0}^{n}$ is lower triangular with diagonal entries \pm 1 . Hence the linear relationship (5.24) is also invertible. Putting together the above observations, it is clear that there is an invertible $(n+1) \times(n+1)$ matrix $\mathcal{C}$ with purely numerical entries $\mathcal{C}_{m, l}$ depending only on $\mu$ and $\nu$ such that

$$
K_{m}\left(\gamma_{0}\right)=\sum_{l=0}^{n} \mathcal{C}_{m, l} H_{l}\left(\gamma_{0}\right)
$$

Since $\gamma_{0}$ is an arbitrary point of the phase space $P(1.2)$, the Lemma follows.

The scattering theoretical idea in the proof the above Lemma goes back to the fundamental works of Moser (see e.g. [8]). However, in the recent paper [14] it has been revitalized in the context of the rational $B C_{n}$ van Diejen model, too. Compared to the rational case, it is a significant difference that our coefficients $\mathcal{U}_{J^{c}, l-|J|}$ (5.16) do depend on the parameters $\mu$ and $\nu$ in a non-trivial manner, whence the observations surrounding the derivations of formula (5.17) turns out to be crucial in our presentation.

Since the family of functions $\left\{H_{l}\right\}_{l=0}^{n}$ Poisson commute, Lemma 14 readily implies that the first integrals $\left\{K_{m}\right\}_{m=0}^{n}$ are also in involution. Now, let us recall that the spectrum of the Lax matrix $L$ is simple, as we have seen in Lemma 4. As a consequence, the eigenvalues of $L$ can be realized as smooth functions of the coefficients of the characteristic polynomial (5.18), thus the following result is immediate.

Theorem 15. The eigenvalues of the Lax matrix $L(3.6)$ are in involution. 
To conclude this section, let us note that the proof of Theorem 15 is quite indirect in the sense that it hinges on the commutativity of the family of functions (5.5). However, the only available proof of this highly non-trivial fact is based on the observation that the Hamiltonians (5.5) can be realized as classical limits of van Diejen's commuting analytic difference operators [1]. As a more elementary approach, let us note that Theorem 15 would also follow from the existence of an $r$-matrix encoding the tensorial Poisson bracket of the Lax matrix $L$ (3.6). Due to Lemma 14, it would imply the commutativity of the family (5.5), too, at least under the specialization (5.8). To find such an $r$-matrix, one may wish to generalize the analogous results on the rational system [15].

\section{Discussion}

One of the most important objects in the study of integrable systems is the Lax representation of the dynamics. By generalizing the earlier results on the rational $B C_{n}$ RSvD models [10, 15, in this paper we succeeded in constructing a Lax pair for the 2-parameter family of hyperbolic van Diejen systems (1.5). Making use of this construction, we showed that the dynamics can be solved by a projection method, which in turn allowed us to initiate the study of the scattering properties of (1.5). Moreover, by combining our scattering theoretical results with the ideas of the recent paper [14], we proved that the first integrals provided by the eigenvalues of the proposed Lax matrix (3.6) are in fact in involution. To sum up, it is fully justified to say that the matrices $L(\underline{3.6})$ and $B$ (4.59) form a Lax pair for the hyperbolic van Diejen system (1.5).

Apart from taking a non-trivial step toward the construction of Lax matrices for the most general hyperbolic van Diejen many-particle systems (5.5), let us not forget about the potential applications of our results. In analogy with the translation invariant RS systems, we expect that the van Diejen models may play a crucial role in clarifying the particle-soliton picture in the context of integrable boundary field theories. While the relationship between the $A$ type RS models and the soliton equations defined on the whole line is under control (see e.g. [4, 17, 27, 28, 29]), the link between the van Diejen models and the soliton systems defined on the half-line is less understood (see e.g. [30, 31]). As in the translation invariant case, the Lax matrices of the van Diejen systems could turn out to be instrumental for elaborating this correspondence.

Turning to the more recent activities surrounding the CMS and the RS many-particle models, let us recall the so-called classical/quantum duality (see e.g. [32, 33, 34, 35, 36]), which relates the spectra of certain quantum spin chains with the Lax matrices of the classical CMS and RS systems. An equally remarkable development is the emergence of new integrable tops based on the Lax matrices of the CMS and the RS systems [37, 38. Relatedly, it would be interesting to see whether the Lax matrix (3.6) of the hyperbolic van Diejen system (1.5) can be fit into these frameworks.

One of the most interesting aspects of the CMS and the RSvD systems we have not addressed in this paper is the so-called Ruijsenaars duality, or action-angle duality. Based on hard analytical techniques, this remarkable property was first exhibited by Ruijsenaars [17] in the context of the translation invariant non-elliptic models. Let us note that in the recent papers [23, 39, 40, 41] almost all of these duality relationships have been successfully reinterpreted in a nice geometrical framework provided by powerful symplectic reduction methods. Moreover, 
by now some duality results are available also for the CMS and the RSvD models associated with the $B C$-type root systems [10, 11, 13].

As for the key player of our paper, we have no doubt that the 2-parameter family of hyperbolic van Diejen systems (1.5) is self-dual. Indeed, upon diagonalizing the Lax matrix $L$ (3.6), we see that the transformed objects defined in (3.24)-(3.25) obey the relationship (3.26), that has the same form as the Ruijsenaars type commutation relation (3.20) we set up in Lemma 3 , Based on the method presented in [17], we expect that the transformed matrix $\hat{L}$ (3.25) shall provide a Lax matrix for the dual system. Therefore, comparing the matrix entries displayed in (3.6) and (3.27), the self-duality of the system (1.5) seems to be more than plausible. Admittedly, many subtle details are still missing for a complete proof. As for filling these gaps, the immediate idea is that either one could mimic Ruijsenaars' scattering theoretical approach, or invent an appropriate symplectic reduction framework. However, notice that the non-standard form of the Hamiltonian (1.5) poses severe analytical difficulties on the study of the scattering theory, whereas the weakness of the geometrical approach lies in the fact that up to now even the translation invariant hyperbolic RS model has not been derived from symplectic reduction. Nevertheless, by taking the analytical continuation of the Lax matrix $L(3.6)$, it is conceivable that the self-duality of the compactified trigonometric version of (1.5) can be proved by adapting the quasi-Hamiltonian reduction approach advocated by Fehér and Klimčík [41]. For further motivation, let us recall that the duality properties are indispensable in the study of the recently introduced integrable random matrix ensembles [42, 43, 44], too.

From the above paragraphs it is clear that our results on the 2-parameter family of hyperbolic systems (1.5) open up a plethora of interesting problems. Besides, based on our numerical calculations, below we also wish to discuss some possible generalizations in two further directions. First, it is a time-honored principle that the inclusion of a spectral parameter into the Lax matrix of an integrable system can greatly enrich the analysis by borrowing techniques from complex geometry. Bearing this fact in mind, with the aid of the function

$$
\Phi(x \mid \eta)=e^{x \operatorname{coth}(\eta)}(\operatorname{coth}(x)-\operatorname{coth}(\eta))
$$

depending on the complex variables $x$ and $\eta$, over the phase space $P$ (1.2) we define the matrix valued smooth function $\mathcal{L}=\mathcal{L}(\lambda, \theta ; \mu, \nu \mid \eta)$ with entries

$$
\left.\mathcal{L}_{k, l}=\left(\mathrm{i} \sin (\mu) F_{k} \bar{F}_{l}+\mathrm{i} \sin (\mu-\nu) C_{k, l}\right)\right) \Phi\left(\mathrm{i} \mu+\Lambda_{j}-\Lambda_{k} \mid \eta\right) \quad\left(k, l \in \mathbb{N}_{N}\right) .
$$

One of the outcomes of our numerical investigations is that for any values of $\eta$ the eigenvalues of $\mathcal{L}$ provide a family of first integrals in involution for the van Diejen system (1.5). Thinking of $\eta$ as a spectral parameter, let us also observe that, in the limit $\mathbb{R} \ni \eta \rightarrow \infty$, from $\mathcal{L}$ we can recover our Lax matrix $L$ (3.6); that is, $\mathcal{L} \rightarrow L$. Although the spectral parameter dependent matrix $\mathcal{L}$ does not take values in the Lie group $U(n, n)(2.3)$, we find it interesting that the constituent function $\Phi$ (6.1) can be seen as a hyperbolic limit of the elliptic Lamé function, that plays a prominent role in the theory of the elliptic CMS and RS systems (see e.g. the papers [45, 5] and the monograph [46]). Therefore, it is tempting to think that an appropriate elliptic deformation of $\mathcal{L}$ (3.6) may lead to a spectral parameter dependent Lax matrix of the elliptic van Diejen system with coupling parameters $\mu$ and $\nu$.

Hitherto we have studied the van Diejen system (1.5) with only two independent coupling parameters. Though a construction of a Lax matrix for the most general hyperbolic van Diejen 
system with five independent coupling parameters still seems to be out of reach, we can offer a plausible conjecture for a Lax matrix with three independent coupling constants. Simply by generalizing the formulae appearing in the theory of the rational $B C_{n} \mathrm{RSvD}$ systems [12, with the aid of an additional real parameter $\kappa$ let us define the real valued functions $\alpha$ and $\beta$ for any $x>0$ by the formulae

$$
\alpha(x)=\frac{1}{\sqrt{2}}\left(1+\left(1+\frac{\sin (\kappa)^{2}}{\sinh (2 x)^{2}}\right)^{\frac{1}{2}}\right)^{\frac{1}{2}} \text { and } \quad \beta(x)=\frac{\mathrm{i}}{\sqrt{2}}\left(-1+\left(1+\frac{\sin (\kappa)^{2}}{\sinh (2 x)^{2}}\right)^{\frac{1}{2}}\right)^{\frac{1}{2}} .
$$

Built upon these functions, let us also introduce the Hermitian $N \times N$ matrix

$$
h(\lambda)=\left[\begin{array}{cc}
\operatorname{diag}\left(\alpha\left(\lambda_{1}\right), \ldots, \alpha\left(\lambda_{n}\right)\right) & \operatorname{diag}\left(\beta\left(\lambda_{1}\right), \ldots, \beta\left(\lambda_{n}\right)\right) \\
-\operatorname{diag}\left(\beta\left(\lambda_{1}\right), \ldots, \beta\left(\lambda_{n}\right)\right) & \operatorname{diag}\left(\alpha\left(\lambda_{1}\right), \ldots, \alpha\left(\lambda_{n}\right)\right)
\end{array}\right]
$$

One can easily show that $h C h=C$, whence the matrix valued function

$$
\tilde{L}=h^{-1} L h^{-1}
$$

also takes values in the Lie group $U(n, n)(2.3)$. Notice that the rational limit of matrix $\tilde{L}$ gives back the Lax matrix of the rational $B C_{n}$ RSvD system, that first appeared in equation (4.51) of paper [11]. Moreover, upon setting

$$
g=\mu, \quad g_{0}=g_{1}=\frac{\nu}{2}, \quad g_{0}^{\prime}=g_{1}^{\prime}=\frac{\kappa}{2},
$$

for van Diejen's main Hamiltonian $H_{1}(\underline{5.5})$ we get that

$$
H_{1}=2 \sum_{a=1}^{n} \cosh \left(\theta_{a}\right) u_{a}\left(1+\frac{\sin (\kappa)^{2}}{\sinh \left(2 \lambda_{a}\right)^{2}}\right)^{\frac{1}{2}}+2 \sum_{a=1}^{n} \operatorname{Re}\left(z_{a} \frac{\sinh \left(\mathrm{i} \kappa+2 \lambda_{a}\right)}{\sinh \left(2 \lambda_{a}\right)}\right)
$$

with the functions $z_{a}$ and $u_{a}$ defined in the equations (3.3) and (3.4), respectively. The point is that, in complete analogy with (5.11), one can establish the relationship

$$
H_{1}+2 \cos (\nu+\kappa+(n-1) \mu) \frac{\sin (n \mu)}{\sin (\mu)}=\operatorname{tr}(\tilde{L}) .
$$

Furthermore, based on numerical calculations for small values of $n$, it appears that the eigenvalues of $\tilde{L}$ (6.5) provide a commuting family of first integrals for the van Diejen system (6.7). To sum up, we have numerous evidences that matrix $\tilde{L}(\underline{6.5})$ is a Lax matrix for the 3-parameter family of van Diejen systems (6.7), if the pertinent parameters are connected by the relationships displayed in (6.6). As can be seen in [11], the new parameter $\kappa$ causes many non-trivial technical difficulties even at the level of the rational van Diejen system. Part of the difficulties can be traced back to the fact that for $\sin (\kappa) \neq 0$ the matrix $\tilde{L}(6.5)$ does not belong to the symmetric space $\exp (\mathfrak{p})(2.9)$, whence the diagonalization of $\tilde{L}$ requires a less direct approach than that provided by the canonical form (2.11). We wish to come back to these problems in later publications. 
Acknowledgments. We are grateful to L. Fehér (Univ. Szeged) for useful comments on the manuscript. The work of B.G.P. was supported by the János Bolyai Research Scholarship of the Hungarian Academy of Sciences, by the Hungarian Scientific Research Fund (OTKA grant K116505), and also by a Lendület Grant; he wishes to thank to Z. Bajnok for hospitality in the MTA Lendület Holographic QFT Group. T.F.G. was supported in part by the Hungarian Scientific Research Fund (OTKA grant K111697) and by COST (European Cooperation in Science and Technology) in COST Action MP1405 QSPACE.

\section{References}

[1] J.F. van Diejen, Commuting difference operators with polynomial eigenfunctions, Compositio Math. 95 (1995) 183-233.

[2] J.F. van Diejen, Deformations of Calogero-Moser systems, Theor. Math. Phys. 99 (1994) 549-554.

[3] J.F. van Diejen, Difference Calogero-Moser systems and finite Toda chains, J. Math. Phys. 36 (1995) 1299-1323.

[4] S.N.M. Ruijsenaars and H. Schneider, A new class of integrable models and its relation to solitons, Ann. Phys. (N.Y.) 170 (1986) 370-405.

[5] S.N.M. Ruijsenaars, Complete integrability of relativistic Calogero-Moser systems and elliptic function identities, Commun. Math. Phys. 110 (1987) 191-213.

[6] F. Calogero, Solution of the one-dimensional $N$-body problem with quadratic and/or inversely quadratic pair potentials, J. Math. Phys. 12 (1971) 419-436.

[7] B. Sutherland, Exact results for a quantum many body problem in one dimension, Phys. Rev. A 4 (1971) 2019-2021.

[8] J. Moser, Three integrable Hamiltonian systems connected with isospectral deformations, Adv. Math. 16 (1975) 197-220.

[9] M.A. Olshanetsky and A.M. Perelomov, Completely integrable Hamiltonian systems connected with semisimple Lie algebras, Invent. Math. 37 (1976) 93-108.

[10] B.G. Pusztai, Action-angle duality between the $C_{n}$-type hyperbolic Sutherland and the rational Ruijsenaars-Schneider-van Diejen models, Nucl. Phys. B 853 (2011) 139-173.

[11] B.G. Pusztai, The hyperbolic $B C_{n}$ Sutherland and the rational $B C_{n}$ Ruijsenaars-Schneider-van Diejen models: Lax matrices and duality, Nucl. Phys. B 856 (2012) 528-551.

[12] B.G. Pusztai, Scattering theory of the hyperbolic $B C_{n}$ Sutherland and the rational $B C_{n}$ Ruijsenaars-Schneider-van Diejen models, Nucl. Phys. B 874 (2013) 647-662.

[13] L. Fehér and T.F. Görbe, Duality between the trigonometric $B C_{n}$ Sutherland system and a completed rational Ruijsenaars-Schneider-van Diejen system, J. Math. Phys. 55 (2014) 102704. 
[14] T.F. Görbe and L. Fehér, Equivalence of two sets of Hamiltonians associated with the rational $B C_{n}$ Ruijsenaars-Schneider-van Diejen system, Phys. Lett. A 379 (2015) 26852689.

[15] B.G. Pusztai, On the classical $r$-matrix structure of the rational $B C_{n}$ Ruijsenaars-Schneider-van Diejen system, Nucl. Phys. B 900 (2015) 115-146.

[16] A.W. Knapp, Lie groups beyond an introduction, Progress in Mathematics, vol. 140, Birkhäuser, Boston, MA, 2002.

[17] S.N.M. Ruijsenaars, Action-angle maps and scattering theory for some finite dimensional integrable systems I. The pure soliton case, Commun. Math. Phys. 115 (1988) 127-165.

[18] R. Abraham and J.E. Marsden, Foundations of Mechanics, second ed., Addison Wesley, 1985.

[19] M.A. Olshanetsky and A.M. Perelomov, Classical integrable finite-dimensional systems related to Lie algebras, Phys. Rep. 71 (1981) 313-400.

[20] D. Kazhdan, B. Kostant and S. Sternberg, Hamiltonian group actions and dynamical systems of Calogero type, Commun. Pure Appl. Math. XXXI (1978) 481-507.

[21] L. Fehér and B.G. Pusztai, Spin Calogero models associated with Riemannian symmetric spaces of negative curvature, Nucl. Phys. B 751 (2006) 436-458.

[22] L. Fehér and B.G. Pusztai, A class of Calogero type reductions of free motion on a simple Lie group, Lett. Math. Phys. 79 (2007) 263-277.

[23] L. Fehér and C. Klimčík, On the duality between the hyperbolic Sutherland and the rational Ruijsenaars-Schneider models, J. Phys. A: Math. Theor. 42 (2009) 185202.

[24] S.N.M. Ruijsenaars, Finite-dimensional soliton systems, in: B. Kupershmidt (Ed.), Integrable and superintegrable systems, World Scientific, 1990, pp. 165-206.

[25] P.P. Kulish, Factorization of the classical and the quantum $S$ matrix and conservation laws, Theor. Math. Phys. 26 (1976) 132-137.

[26] J. Moser, The scattering problem for some particle systems on the line, in: Lecture Notes in Mathematics 597, Springer, 1977, pp. 441-463.

[27] O. Babelon and D. Bernard, The sine-Gordon solitons as an N-body problem, Phys. Lett. B 317 (1993) 363-368.

[28] S.N.M. Ruijsenaars, Action-angle maps and scattering theory for some finite dimensional integrable systems II. Solitons, antisolitons and their bound states, Publ. RIMS 30 (1994) 865-1008.

[29] S.N.M. Ruijsenaars, Action-angle maps and scattering theory for some finite dimensional integrable systems III. Sutherland type systems and their duals, Publ. RIMS 31 (1995) 247-353. 
[30] H. Saleur, S. Skorik and N.P. Warner, The boundary sine-Gordon theory: classical and semi-classical analysis, Nucl. Phys. B 441 (1995) 421-436.

[31] A. Kapustin and S. Skorik, On the non-relativistic limit of the quantum sine-Gordon model with integrable boundary condition, Phys. Lett. A 196 (1994) 47-51.

[32] E. Mukhin, V. Tarasov and A. Varchenko, Gaudin Hamiltonians generate the Bethe algebra of a tensor power of the vector representation of $\mathfrak{g l}_{N}$, St. Petersburg Math. J. 22 (2011) 463-472.

[33] A. Alexandrov, S. Leurent, Z. Tsuboi and A. Zabrodin, The master T-operator for the Gaudin model and the KP hierarchy, Nucl. Phys. B 883 (2014) 173-223.

[34] A. Gorsky, A. Zabrodin and A. Zotov, Spectrum of Quantum Transfer Matrices via Classical Many-Body Systems, JHEP 01 (2014) 070.

[35] Z. Tsuboi, A. Zabrodin and A. Zotov, Supersymmetric quantum spin chains and classical integrable systems, JHEP 05 (2015) 086.

[36] M. Beketov, A. Liashyk, A. Zabrodin and A. Zotov, Trigonometric version of quantumclassical duality in integrable systems, Nucl. Phys. B 903 (2016) 150-163.

[37] G. Aminov, S. Arthamonov, A. Smirnov and A. Zotov, Rational Top and its Classical R-matrix, J. Phys. A: Math. Theor. 47 (2014) 305207.

[38] A. Levin, M. Olshanetsky and A. Zotov, Relativistic Classical Integrable Tops and Quantum R-matrices, JHEP 07 (2014) 012.

[39] L. Fehér and V. Ayadi, Trigonometric Sutherland systems and their Ruijsenaars duals from symplectic reduction, J. Math. Phys. 51 (2010) 103511.

[40] L. Fehér and C. Klimčík, Poisson-Lie interpretation of trigonometric Ruijsenaars duality, Commun. Math. Phys. 301 (2011) 55-104.

[41] L. Fehér and C. Klimčík, Self-duality of the compactified Ruijsenaars-Schneider system from quasi-Hamiltonian reductions, Nucl. Phys. B 860 (2012) 464-515.

[42] E. Bogomolny, O. Giraud and C. Schmit, Random Matrix Ensembles Associated with Lax Matrices, Phys. Rev. Lett. 103 (2009) 054103.

[43] E. Bogomolny, O. Giraud and C. Schmit, Integrable random matrix ensembles, Nonlinearity 24 (2011) 3179-3213.

[44] Y.V. Fyodorov and O. Giraud, High values of disorder-generated multifractals and logarithmically correlated processes, Chaos, Solitons \& Fractals 74 (2015) 15-26.

[45] I.M. Krichever, Elliptic solutions of the Kadomtsev-Petviashvili equation and integrable systems of particles, Funct. Anal. Appl. 14 (1980) 282-290.

[46] O. Babelon, D. Bernard and M. Talon, Introduction to classical integrable systems, Cambridge University Press, 2003. 